\title{
DIVISORS OF A MODULE AND BLOW UP
}

\author{
ANA L. BRANCO CORREIA AND SANTIAGO ZARZUELA
}

\begin{abstract}
In this paper we work with several divisors of a module $E \subseteq G \simeq R^{e}$ having rank $e$, such as the classical Fitting ideals of $E$ and of $G / E$, and the more recently introduced (generic) Bourbaki ideals $I(E)$ (A. Simis, B. Ulrich, and W. Vasconcelos [18]) or ideal norms $[[E]]_{R}(\mathrm{O}$. Villamayor [22]). We found several relations and equalities among them which allow to describe in some cases universal properties with respect to $E$ of their blow ups. As a byproduct we are also able to obtain lower bounds for the analytic spread $\ell\left(\bigwedge^{e} E\right)$, related with the algebraic local version of Zak's inequality as explained in A. Simis, K. Smith and B. Ulrich [16].
\end{abstract}

\section{INTRODUCTION}

Paraphrasing W. Vasconcelos [21], the divisors of a module $E$ over a ring $R$ are the ideals of $R$ that carry important information about the structure and properties of $E$. Let $E$ be a finitely generated torsionfree $R$-module having rank $e$ with a fixed embedding $f: E \subseteq G \simeq R^{e}$. Then the Fitting ideals of $E$ and of $G / E$ are typical examples. They determine the free locus, and they give rise in particular to the notions of principal class, equimultiple, complete intersection, etc. Another divisor ideal that play a significant role in the study of the integral closure of $\mathrm{E}$ is $\operatorname{det}_{f}(E)$, introduced by Rees in [14]. By definition $\operatorname{det}_{f}(E)$ is the ideal defined by the image of the mapping $\bigwedge^{e} f$ in $\bigwedge^{e} R^{e} \simeq R$ and up to isomorphism $\operatorname{det}_{f}(E)=\bigwedge^{e} E / \tau_{R}\left(\bigwedge^{e} R^{e}\right)$. In [21] the ideal $\operatorname{det}_{f}(E)$ is also denoted by $\operatorname{det}_{0}(E)$, since it does not depend on $f$. It is useful for instance to lead with reductions of modules. In fact, if $U \subseteq E$ are modules of the same rank over a domain, then $U$ is a reduction of $E$ if and only if $\operatorname{det}_{0}(U)$ is a reduction of $\operatorname{det}_{0}(E)$. See also [19] or [21] for further details.

On the other hand, O. Villamayor attached in 22 a new class of ideals to $E$, called the norm of $E$ and denoted by $[[E]]_{R}$, and showed that the blow up at any representant of this class has a universal flattening property. That is, if $X \stackrel{\pi}{\longrightarrow}$ $\operatorname{Spec}(R)$ is the blow up of $R$ with center $[[E]]_{R}$, then $\pi^{*}(E) / \operatorname{tor}\left(\pi^{*}(E)\right)$ is a locally free sheaf of $\mathcal{O}_{X}$-modules of rank $e$, and $\pi$ is universal for this property. He named this blow up as the blow up of $R$ at $E$. In the analytical setting the construction of

Date: June 16, 2018.

Key words and phrases. Divisors of a module, Blow up, Zak's inequality, ideal modules, generic Bourbaki ideals.

The research of the first author was made within the activities of the Centro de Estruturas Lineares e Combinatrias and was partially supported by the Fundação para a Ciência e Tecnologia (Strategic Project PEst-OE/MAT/UI1431/2011).

The second author was supported by MTM2010-20279-C02-01. 
a homomorphism with such flattening property was established by H. Rossi in [15], who asked for the determination of this construction by some universal property. In fact, under the name of Nash transformation of $R$ at $E$ the existence in general of this universal construction was established by A. Oneto and E. Zatini in [12], obtaining as a particular case of this construction the usual Nash blowing-up. Similarly, the higher Nash blowups defined by T. Yasuda in [23] may be seen as particular cases of these general Nash transformations. And also the $e$-th $F$-blow ups, $e$ a non-negative integer, of a variety $X$ of positive characteristic as defined by Yasuda himself in [24].

The main novelty in Villamayor's construction is that he showed that there exists a module $E_{1}$ of projective dimension at most 1 having rank $e$ whose Fitting ideal $F_{e}\left(E_{1}\right)$ is a representative of $[[E]]_{R}$, as a fractional ideal. This Fitting ideal $F_{e}\left(E_{1}\right)$ is in fact a sub-determinantal ideal of any matrix representing $E$, which gives an effective method to compute the blow up of $R$ at $E$. For instance, N. Hara, T. Sawada and T. Yasuda have recently used this approach in [5] in order to compute explicitly $F$-blowups for some surface singularities by using Macaulay2 [10].

In section 2, we make clear the analogies of these ideals by showing that $\operatorname{det}_{0}(E)=$ $F_{0}(G / E)$ is also a representant of $[[E]]_{R}$. To do this we explore the relationship between Fitting ideals and determinant ideals with exterior algebras. Moreover, by using that $\operatorname{det}_{0}(E) \operatorname{det}_{0}(E)^{-1}$ defines the non-free locus of $E$ and that $\operatorname{det}_{0}(E) \cdot S$ is invertible if and only if $E \otimes_{R} S / \tau_{R}\left(E \otimes_{R} S\right)$ is free for any birational extension $S$ of $R$, we may also show that the blow of $R$ at $\operatorname{det}_{0}(E)$ has the universal flattening property, providing another proof for the existence of this universal object, somehow in the spirit of [12, section 2].

As a byproduct of the above study we get some results concerning the algebraic local version of the so-called Zak's inequality. Let $R$ be a Noetherian ring and $E$ a finitely generated torsionfree $R$-module having a rank. We define the Rees algebra $\mathcal{R}(E)$ of $E$ to be the quotient $\mathcal{S}(E) / \tau_{R}(\mathcal{S}(E)$ ) of the symmetric algebra by its $R$-torsion submodule $\tau_{R}(\mathcal{S}(E))$. $\mathcal{R}(E)$ inherits a natural graduation from the symmetric algebra and we denote by $E^{n}$ the $n$th graded piece, that is, $E^{n}:=\mathcal{R}(E)_{n}$. If $(R, \mathfrak{m}, k)$ is also a local ring the fiber cone of $\mathcal{R}(E)$ is the graded ring $\mathcal{F}(E):=$ $\mathcal{R}(E) \otimes_{R} k=\bigoplus_{n \geq 0} E^{n} / \mathfrak{m} E^{n}$. The Krull dimension of $\mathcal{F}(E)$ is called the analytic spread of $E$ and is denoted by $\ell(E)$. In [17], A. Simis and B. Ulrich discussed the problem

$$
\text { "when does the inequality } \ell\left(\bigwedge^{e} E\right) \geq \text { ht } F_{e}(E) \text { hold?" }
$$

posed in [16]. This problem is related to the named Zak's inequality for the dimension of the image of the Gauss map when the variety is not smooth in terms of the dimension of its singular locus. Using the relationships obtained in section 2 for the considered divisor ideals, we also give some affirmative answers for this question (cf. Proposition 2.3 and Corollary 2.5).

For some modules $E$ it is possible to find a free submodule $F$ such that the quotient $E / F$ is isomorphic to an ideal $I$. For example this happens whenever $R$ is a Noetherian normal domain and $E$ is a torsionfree $R$-module (cf. [1, Chap. 7, $\S 4$, Théorème 6]). If it exists, the ideal $I \simeq E / F$ is called a Bourbaki ideal of $E$. More 
generally an exact sequence of $R$-modules

$$
0 \rightarrow F \rightarrow E \rightarrow I \rightarrow 0
$$

with $F$ a free module and $I$ an $R$-ideal is called a Bourbaki sequence. In [18], A. Simis, B. Ulrich, and W. Vasconcelos developed the notion of generic Bourbaki ideals defined over a suitable faithfully flat extension of $R$. This approach has the advantage that the Bourbaki ideal thus obtained is essentially unique. As a matter of fact, they proved that for some families of modules $E$ over a local ring $R$ and having rank there is a suitable Nagata extension $R^{\prime \prime}$ of $R$, together with a free $R^{\prime \prime}$-module $F$ such that $E^{\prime \prime} / F=\bar{E}$ is isomorphic to an $R^{\prime \prime}$-ideal of rank 1 , where $E^{\prime \prime}=E \otimes_{R} R^{\prime \prime}$ (cf. [18, Proposition 3.2]). They wrote $I(E)$ to denote this ideal and called it a generic Bourbaki ideal of $E$. The induced epimorphism of $R^{\prime \prime}$-algebras $\mathcal{R}(E) \otimes_{R} R^{\prime \prime} \simeq \mathcal{R}\left(E^{\prime \prime}\right) \rightarrow \mathcal{R}(I(E))$ plays a major role throughout their work. They used it to transfer properties about $\mathcal{R}(E)$ to $\mathcal{R}(I(E))$ and vice-versa. In fact, in the case where the kernel is generated by a regular sequence on $\mathcal{R}\left(E^{\prime \prime}\right)$, then $\mathcal{R}\left(E^{\prime \prime}\right)$ is a deformation of $\mathcal{R}(I(E))$ and properties such as Cohen-Macaulayness and normality can be transferred from $\mathcal{R}(I(E))$ to $\mathcal{R}\left(E^{\prime \prime}\right)$, and hence to $\mathcal{R}(E)$ (cf. [18, Theorem 3.5]).

In 4] we examined their construction of $I(E)$ and proved other properties. In particular, we related the depth of $\mathcal{R}(E)$ and of $\mathcal{R}(I(E))$. In section 4 we establish other relations between $E$ and a $I(E)$. Especially, we obtain a formula relating the minimal number of generators of $E$ and of $I(E)$.

Theorem. [cf. Theorem 4.4 Let $R$ be a Noetherian local ring, let $E$ be a finitely generated $R$-module having rank $e \geq 2$ (and $U$ a reduction of $E$ ). If $I(E)$ is a generic Bourbaki ideal of $E$ (with respect to $U$ ) then

$$
\mu(E)=\mu(I(E))+e-1 .
$$

This formula may be seen as an extension of a similar one for the analytic spread proved in [18]. In particular, Eq. (1.1) implies that there exists a generic Bourbaki $I(E)$ which is perfect of grade 2 if and only if $E$ is not a free $R$-module and also proj $\operatorname{dim} E=1$.

In section 5, we relate generic Bourbaki ideals with Fitting ideals for a finitely generated $R$-module $E$ having rank $e \geq 2$. Supposing that $I \simeq E^{\prime \prime} / F$ is a generic Bourbaki ideal of $E$ over a Nagata extension $R^{\prime \prime}$ of $R$, we extend a basis $x_{1}, \ldots, x_{e-1}$ of $F$ to a generating set $x_{1}, \ldots, x_{n}$ of $E^{\prime \prime}=E \otimes_{R} R^{\prime \prime}$ and consider a finite free presentation $R^{\prime \prime m} \stackrel{\varphi}{\rightarrow} R^{\prime \prime n} \stackrel{\phi}{\rightarrow} E^{\prime \prime} \rightarrow 0$ with respect to this set of generators, that is $\left[\begin{array}{lll}x_{1} & \cdots & x_{n}\end{array}\right] \varphi=0$. For this presentation there exists an $(n-e+1) \times(n-e)$ submatrix $\psi$ of $\varphi$ satisfying grade $I_{n-e}(\psi) \geq 1$, and in the case where grade $F_{e}(E) \geq$ 2 then $E^{\prime \prime} / F \simeq I_{n-e}(\psi)$ (see Theorem 5.3). As a consequence, we deduce that any generic Bourbaki ideal is always isomorphic to a Fitting ideal. It is important to note this Fitting ideal is also a sub-determinantal ideal of a specific presentation of $E^{\prime \prime}$.

The interaction between their Rees algebras and the relations between numerical invariants of $E$ and $I(E)$ such as reduction number, analytic spread, minimal number 
of generators, justify that a generic Bourbaki ideal $I(E)$ may also be viewed as a divisor of $E$. In the final section $[6$ we return to the questions about the relations among the different divisor ideals of $E$ including now the generic Bourbaki ideal. The realization obtained by Villamayor of the norm ideal as a Fitting ideal is the key for that inclusion. Observing first that the norm ideal behaves well under the extension of scalars by flat homomorphisms, we have that

$$
I(E) \simeq I_{n-e}(\psi) \subseteq I_{n-e}(\rho) \subseteq F_{e}\left(E^{\prime \prime}\right),
$$

with $\rho$ the $n \times(n-e)$ submatrix of $\varphi$ containing $\phi$ and $I_{n-e}(\rho) \simeq\left[\left[E^{\prime \prime}\right]\right]_{R^{\prime \prime}} \simeq$ $\operatorname{det}_{0}(E) \cdot R^{\prime \prime}$. If moreover proj $\operatorname{dim} E=1$ then $I_{n-e}(\rho)=F_{e}\left(E^{\prime \prime}\right)$. In particular, since if $E$ is a contracted module over a 2-dimensional regular local ring it may be seen that $F_{e}\left(E^{\prime \prime}\right)$ itself is a generic Bourbaki ideal [6], we get that there exists a generic Bourbaki ideal $I(E)$ of $E$ which is a representative of $\left[\left[E^{\prime \prime}\right]\right]_{R^{\prime \prime}}$ (cf. Corollary 6.3), and so it fulfills the corresponding universal flattening property with respect to $E^{\prime \prime}$.

For any unexplained terminology we refer to the book of W. Bruns and J. Herzog [2]. Finally, we would like to thank T. Yasuda for telling us about the paper by Oneto-Zatini [12].

\section{Divisors of A MOdule - PART 1}

2.1. Fitting ideals. Let $E$ be a finitely generated $R$-module. Then the Fitting ideals of $E$ are typical examples of divisors of $E$. By definition, $F_{i}(E):=I_{n-i}(\varphi)$ is the ideal generated by the $(n-i) \times(n-i)$ minors of $\varphi$ where

$$
R^{m} \stackrel{\varphi}{\rightarrow} R^{n} \stackrel{\phi}{\rightarrow} E \rightarrow 0
$$

is a finite presentation of $E, 0 \leq i \leq n$. This ideal is independent of the choices of the generators and of any finite free presentation (2.1) of $E$. We have that $F_{i}(E) \subseteq$ $F_{i+1}(E)$ and if, in addition, $E$ has rank $e$ (which means that $E \otimes_{R} \operatorname{Quot}(R) \simeq$ Quot $\left.(R)^{e}\right)$ then $F_{e}(E)$ is the smallest Fitting ideal which is different from zero. Hence if $E$ has positive rank $e$ then

$$
F_{0}(E)=\cdots=F_{e-1}(E)=(0) \subsetneq F_{e}(E) \subseteq \cdots \subseteq F_{i}(E) \subseteq \cdots \subseteq R .
$$

Now suppose that we have a given embedding $E \subseteq G \simeq R^{e}$ with $E$ of rank $e$. Then, $G / E$ has rank 0 and the first non-zero Fitting ideals $F_{e}(E)$ and $F_{0}(G / E)$ are related by the inclusion $V\left(F_{e}(E)\right) \subseteq V\left(F_{0}(G / E)\right)=\operatorname{Supp} G / E$. Recall that a torsionfree module $E \subseteq G \simeq R^{e}$ is an ideal module if grade $G / E \geq 2$. (This condition implies that $E$ has rank $e$. Also that $E$ is not free if $E \subsetneq G$.) In this case,

$$
V\left(F_{e}(E)\right)=V\left(F_{0}(G / E)\right)=\operatorname{Supp} G / E .
$$

In particular, grade $F_{e}(E) \geq 2$. See [3, section 3] for further details. In this section, we prove that $F_{0}(G / E) \subseteq F_{e}(E)$ (up to an isomorphism), and that the equality holds for ideal modules having projective dimension equal to one (see Proposition 2.6). 
2.2. Determinants. Let $E$ be a finitely generated $R$-module and let $f: E \rightarrow R^{e}$ be an $R$-homomorphim. The image of the mapping $\bigwedge^{e} f$ in $\bigwedge^{e} R^{e} \simeq R$ defines an $R$-ideal, that is $\operatorname{im} \bigwedge^{e} f=I \cdot \bigwedge^{e} R^{e} \subset \bigwedge^{e} R^{e}$, where $I$ is an $R$-ideal, and we write (abusing notation slightly)

$$
\operatorname{det}_{f}(E):=I=\operatorname{im} \bigwedge^{e} f \subseteq R .
$$

This ideal is called the determinant of the module $E$ with respect to $f$.

The module $E$ does not need to be torsionfree. In fact, since $\tau_{R}(E) \subseteq \operatorname{ker} f$, we always have a commutative diagram

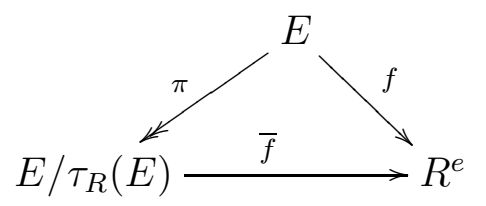

where $\pi$ is the canonical epimorphism and $\bar{f} \circ \pi=f$. We have the following properties.

Properties 2.1. Let $R$ be a Noetherian ring. Let $E$ be a finitely generated $R$-module, $f: E \rightarrow R^{e}$ an $R$-homomorphism and $\varphi: R \rightarrow S$ a homomorphism of rings. Then

a) $\operatorname{det}_{f}(E)=\operatorname{det}_{\bar{f}}\left(E / \tau_{R}(E)\right)$.

b) $\operatorname{det}_{f \otimes \mathrm{id}}\left(E \otimes_{R} S\right)=\operatorname{det}_{f}(E) \cdot S$.

c) $\operatorname{det}_{f \otimes \mathrm{id}}\left(E \otimes_{R} S\right)=\operatorname{det}_{f \otimes \mathrm{id}}\left(E \otimes_{R} S / \tau_{S}\left(E \otimes_{R} S\right)\right)$.

Proof. (a) $\bigwedge^{e} \pi: \bigwedge^{e} E \rightarrow \bigwedge^{e} E / \tau_{R}(E)$ is an epimorphism, hence im $\bigwedge^{e} f=\operatorname{im} \bigwedge^{e} \bar{f}$ and (a) follows.

(b) The homomorphism $f$ induces the commutative diagram

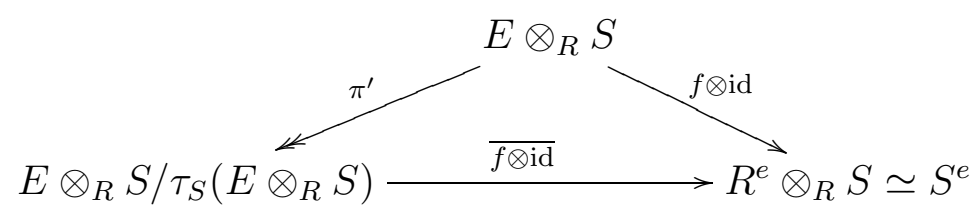

The exterior powers commute with base extensions, and so applying $\bigwedge^{e}$ we get commutative diagrams

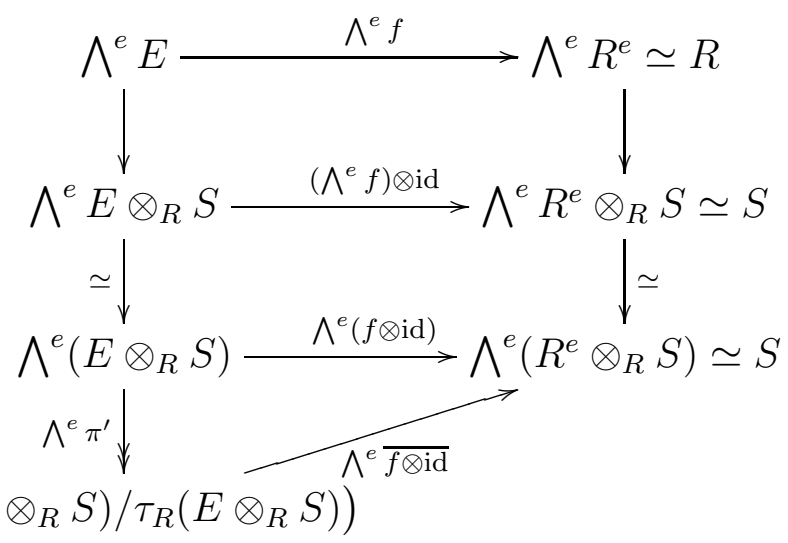


Now

$$
\operatorname{det}_{f \otimes i d}\left(E \otimes_{R} S\right)=\operatorname{im} \bigwedge^{e}(f \otimes \mathrm{id})=\left(\operatorname{im} \bigwedge^{e} f\right) \cdot S=\operatorname{det}_{f}(E) \cdot S .
$$

(c) follows by (a) and (b).

We say that $f$ has rank if im $f$ has rank. In this case, also ker $f \subset E$ has rank. Assume that $E$ and $f$ have both rank $e$ and put $Q=\operatorname{Quot}(R)$ for the total ring of fractions of $R$. Then, $\operatorname{rank}(f \otimes \mathrm{id})=e$ and $f \otimes \mathrm{id}: E \otimes_{R} Q \longrightarrow R^{e} \otimes_{R} Q$ is an isomorphism. Moreover, the homomorphism $\bar{f}: E / \tau_{R}(E) \rightarrow R^{e}$ is injective. This is a consequence of the following observation.

Lemma 2.2. Let $R$ be a Noetherian ring. Let $E$ be a finitely generated torsionfree $R$-module having rank $e$ and $f: E \rightarrow R^{e}$ an $R$-homomorphism with rank $f=e$. Then $f$ is a monomorphism.

Proof. We have that $E \otimes_{R} Q \simeq f(E) \otimes_{R} Q \simeq Q^{e}$ and so ker $f \otimes_{R} Q=0$. This implies that ker $f \subseteq \tau_{R}(E)=0$, proving that $f$ is a monomorphism.

2.3. Determinants and Fitting ideals. In order to see determinants as Fitting ideals we fix some notation. For a positive integer $n$, we set $[n]=\{1, \ldots, n\}$. Let $H=\left\{i_{1}, \ldots, i_{h}\right\}$ be a subset of $[n]$ and suppose that $i_{1}<i_{2}<\cdots<i_{h}$. We write

$$
x_{H}=x_{i_{1}} \wedge x_{i_{2}} \wedge \cdots \wedge x_{i_{h}} .
$$

Let $A=\left(\alpha_{i j}\right)$ be an $n \times m$ matrix over a ring $R$. For subsets $\left\{i_{1}<\cdots<i_{r}\right\} \subseteq[n]$ and $\left\{j_{1}<\cdots<j_{s}\right\} \subseteq[m]$ we write

$$
A\left[i_{1}, \ldots, i_{r} \mid j_{1}, \ldots, j_{s}\right]=\left[\begin{array}{ccc}
\alpha_{i_{1}, j_{1}} & \cdots & \alpha_{i_{1}, j_{s}} \\
\vdots & & \vdots \\
\alpha_{i_{r}, j_{1}} & \cdots & \alpha_{i_{r}, j_{s}}
\end{array}\right] .
$$

Suppose that $E$ is generated by $x_{1}, \ldots, x_{n}$. Suppose $m \leq n$. Hence $\left\{x_{H}: H \in\right.$ $\left.\mathcal{P}_{m}([n])\right\}$, where $\mathcal{P}_{m}([n])$ denotes the set of all subsets of $[n]$ with $m$ elements, is the corresponding generating set of $\bigwedge^{m} E$. If $u_{j}=\sum_{i=1}^{n} \alpha_{i j} x_{i}$ for $1 \leq j \leq m$, then

$$
u_{1} \wedge \cdots \wedge u_{m}=\sum_{H=\left\{i_{1}<\cdots<i_{m}\right\} \in \mathcal{P}_{m}([n])} \operatorname{det} A\left[i_{1}, \ldots, i_{m} \mid 1, \ldots, m\right] x_{H} .
$$

Now, let $\left(v_{1}, \ldots, v_{e}\right)$ be any basis of $R^{e}$. Hence $\bigwedge^{e} R^{e}=\left\langle v_{1} \wedge \cdots \wedge v_{e}\right\rangle \simeq R$. Let $f: E \rightarrow R^{e}$ be an $R$-homomorphism and suppose that $f\left(x_{i_{j}}\right)=\sum_{k=1}^{e} \alpha_{k j} v_{k}$ and put $A=\left(\alpha_{i j}\right)$. Therefore

$$
\begin{aligned}
\operatorname{im} \wedge^{e} f & =\left\langle f\left(x_{i_{1}}\right) \wedge f\left(x_{i_{2}}\right) \wedge \cdots \wedge f\left(x_{i_{e}}\right): i_{1}<i_{2}<\cdots<i_{e}\right\rangle \\
& =\left\langle\operatorname{det} A\left[i_{1}, \ldots, i_{e} \mid 1, \ldots, e\right] v_{1} \wedge \cdots \wedge v_{e}: i_{1}<i_{2}<\cdots<i_{e}\right\rangle \\
& =\left\langle\operatorname{det} A\left[i_{1}, \ldots, i_{e} \mid 1, \ldots, e\right]: i_{1}<i_{2}<\cdots<i_{e}\right\rangle \cdot \wedge^{e} R^{e} .
\end{aligned}
$$

It follows that $\operatorname{det}_{f}(E)$ is the ideal generated by the $e \times e$ minors of $A=\left(\alpha_{i j}\right)$. Moreover, since $E$ is generated by $n$ elements, we have a natural epimomorphism $R^{n} \rightarrow E$. Hence

$$
R^{n} \underset{\psi}{\longrightarrow} \stackrel{f}{\longrightarrow} R^{e}=G \longrightarrow G / \operatorname{im} f
$$


is a finite presentation of $G / \operatorname{im} f$. Therefore $F_{0}(G / \operatorname{im} f)=I_{e}(\psi)$ and we have

$$
\operatorname{det}_{f}(E)=F_{0}(G / \operatorname{im} f) .
$$

Now assume that $E$ has rank $e$ and let $Q=\operatorname{Quot}(R)$ be the total ring of fractions of $R$. Hence we have natural homomorphisms

$$
\bigwedge^{e} E \rightarrow\left(\bigwedge^{e} E\right) \otimes_{R} Q \simeq \bigwedge^{e}\left(E \otimes_{R} Q\right) \simeq \bigwedge^{e} Q^{e} \simeq Q
$$

Suppose also that $f: E \rightarrow R^{e}$ is an $R$-homomorphism such that $\operatorname{im} f$ has $\operatorname{rank} e$. (Note that if $E$ has rank $e$ one can always find such an $R$-homomorphism $f$. Also, that this is the case if $f: E \hookrightarrow R^{e}$ is an embedding.) Hence we have a natural Q-isomorphism

$$
Q^{e} \simeq E \otimes_{R} Q \stackrel{f \otimes \mathrm{id}}{\longrightarrow} R^{e} \otimes_{R} Q \simeq Q^{e}
$$

and natural homomorphisms

$$
\bigwedge^{e} E \stackrel{\Lambda^{e} f}{\longrightarrow} \bigwedge^{e} R^{e} \hookrightarrow\left(\bigwedge^{e} R^{e}\right) \otimes_{R} Q \simeq \bigwedge^{e}\left(R^{e} \otimes_{R} Q\right) \simeq \bigwedge^{e} Q^{e} \simeq Q .
$$

Therefore, we get commutative diagrams

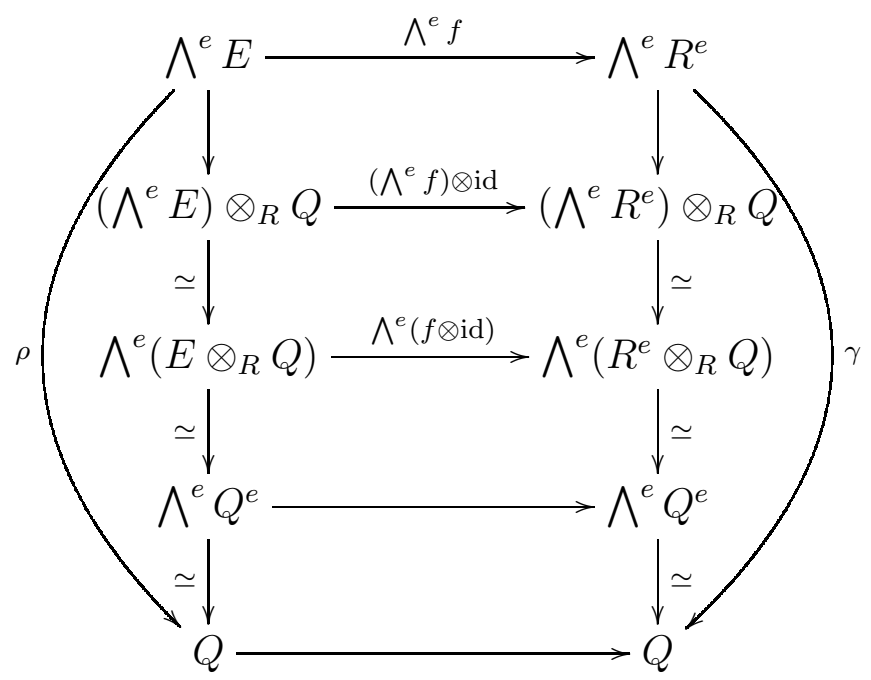

where $\left(\bigwedge^{e} f\right) \otimes$ id is an isomorphism. This implies that $\operatorname{ker}\left(\bigwedge^{e} f\right) \subseteq \tau_{R}\left(\bigwedge^{e} E\right)$. Moreover, $\bigwedge^{e} R^{e}$ is torsionfree and so $\operatorname{ker}\left(\bigwedge^{e} f\right)=\tau_{R}\left(\bigwedge^{e} E\right)$ is independent of $f$. Therefore one has

$$
\operatorname{det}_{f}(E) \simeq \bigwedge^{e} E / \tau_{R}\left(\bigwedge^{e} E\right) .
$$

This independence allow us to forget the homomorphism $f$. In fact, in [21] the ideal $\operatorname{det}_{f}(E)$ is denoted by $\operatorname{det}_{0}(E)$. This ideal is, then, an invariant of $E$, called the order determinant of $E$, and we have

$$
\operatorname{det}_{0}(E):=\operatorname{det}_{f}(E),
$$

where $f: E \rightarrow R^{e}$ is an $R$-homomorphism with $\operatorname{rank} f=e$. Therefore

$$
\operatorname{det}_{0}(E)=\operatorname{det}_{f}(E)=F_{0}(G / \operatorname{im} f)=\operatorname{im} \bigwedge^{e} f \simeq \bigwedge^{e} E / \tau_{R}\left(\bigwedge^{e} E\right) .
$$

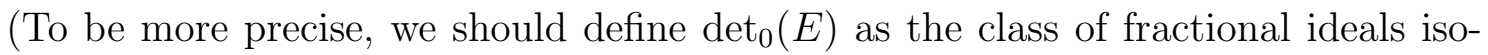
morphic to $\bigwedge^{e} E / \tau_{R}\left(\bigwedge^{e} E\right)$, but for the purposes in this paper we prefer to think 
$\operatorname{det}_{0}(E)$ as any ideal of $R$ in this class of the form $\operatorname{det}_{f}(E)$, with $f$ of rank $e$. Note that since $\operatorname{det}_{f}(E)=I_{e}(\psi)$ with $\operatorname{rank} \psi=e$, then $\operatorname{det}_{f}(E)$ has positive grade.)

Assume that $E$ is a finitely generated torsionfree $R$-module having rank $e>0$. The modules $\bigwedge^{e} E$ and $\bigwedge^{e} E / \tau_{R}\left(\bigwedge^{e} E\right)$ have the same Rees algebra. Therefore, the above approach allows us, by means of the equalities in Eq. (2.9), to deduce a lower bound for the analytic spread $\ell\left(\bigwedge^{e} E\right)$. In particular, we obtain an affirmative answer for ideal modules to the question posed in [17]. We note that ideal modules are orientable (which means that $\left(\bigwedge^{e} E\right)^{* *} \simeq R$ ); in this case an affirmative answer is given in [17, Corollary 3.2] under the assumption that $R$ is a local equidimensional and universally catenary ring.

Proposition 2.3. Let $R$ be a Noetherian local ring and $E \subseteq G \simeq R^{e}$ a finitely generated torsionfree $R$-module having rank $e>0$, but not free. Then

$$
\ell\left(\bigwedge^{e} E\right) \geq \operatorname{ht} F_{0}(G / E)
$$

If $E$ is an ideal module then

$$
\ell\left(\bigwedge^{e} E\right) \geq \operatorname{ht} F_{e}(E) .
$$

Proof. In this case $\operatorname{im} f=E$ and we have

$$
\begin{aligned}
\ell\left(\bigwedge^{e} E\right) & =\ell\left(\bigwedge^{e} E / \tau_{R}\left(\bigwedge^{e} E\right)\right)=\ell\left(\operatorname{det}_{0}(E)\right)=\ell\left(F_{0}(G / E)\right) \\
& \geq \operatorname{ht}\left(F_{0}(G / E)\right)=\operatorname{ht} F_{e}(E)
\end{aligned}
$$

- the last equality holds in the case where $E$ is an ideal module (by (2.2) ).

2.4. The norm of a module. Let $E$ be a finitely generated $R$-module having rank $e$. O. Villamayor attached in [22] a class of ideals to $E$ called the norm of $E$ and denoted by $[[E]]_{R}$, and showed that the blow up at any representant of this class has a universal flattening property. According to [22]

$$
[[E]]_{R}:=\operatorname{im}\left(\bigwedge^{e} E \rightarrow Q \simeq \bigwedge^{e} E \otimes_{R} Q\right)
$$

and, any fractional ideal isomorphic to this one is a representative of $[[E]]_{R}$. In particular, $[[E]]_{R}=\operatorname{im} \rho$, where $\rho$ is as in the diagram (2.6). Moreover, since $\operatorname{im} \rho \simeq \gamma\left(\operatorname{im} \bigwedge^{e} f\right)$ then considered $\operatorname{det}_{0}(E)$ as fractional ideal we get

$$
[[E]]_{R} \simeq \operatorname{det}_{0}(E) .
$$

Let $X=\operatorname{Spec}(R)$. Then the blow up of $X$ at $E$, denoted by $\mathrm{Bl}_{E}(X)$, is defined as the blow up of $X$ with respect to any representative of $[[E]]_{R}$. In particular, we have that

$$
\mathrm{Bl}_{E}(X) \simeq \operatorname{Proj} \mathcal{R}\left[\operatorname{det}_{0}(E) t\right]=\operatorname{Proj}\left(\bigoplus_{n \geq 0} \operatorname{det}_{0}(E)^{n} t^{n}\right)
$$

The following construction is done in [22] to determine another ideal representing $[[E]]_{R}$ : Since $E$ has rank $e$ we can choose an $n \times(n-e)$ submatrix $\varphi^{\prime}$ of $\varphi$, in (2.1), with rank $(n-e)$, that is having a non-zero divisor $(n-e) \times(n-e)$ minor. Hence there exists a free $R$-submodule $M$ of ker $\phi$ having rank $(n-e)$. The module $E_{1}:=R^{n} / M=\operatorname{coker}\left(R^{n-e} \stackrel{\varphi^{\prime}}{\rightarrow} R^{n}\right)$ is an $R$-module with a finite free presentation 
$0 \rightarrow R^{n-e} \rightarrow R^{n} \rightarrow E_{1} \rightarrow 0$. Moreover, the module $E_{1}$ has rank $e$, there is a natural surjection $E_{1} \stackrel{\nu}{\rightarrow} E$ and $E_{1} / \tau_{R}\left(E_{1}\right) \simeq E / \tau_{R}(E)$. Further, by Properties 2.1,

$$
[[E]]_{R} \simeq \operatorname{det}_{0}(E)=\operatorname{det}_{0}\left(E / \tau_{R}(E)\right)=\operatorname{det}_{0}\left(E_{1} / \tau_{R}\left(E_{1}\right)\right)=\operatorname{det}_{0}\left(E_{1}\right) \simeq\left[\left[E_{1}\right]\right]_{R} .
$$

Therefore, the blow ups at $E$ and $E_{1}$ are isomorphic. Moreover, by [22, Proposition 2.5], the Fitting ideal $F_{e}\left(E_{1}\right)$ is a representative of $\left[\left[E_{1}\right]\right]_{R}$ as a fractional ideal. Hence

$$
\mathrm{Bl}_{E}(X) \simeq \operatorname{Proj} \mathcal{R}\left[F_{e}\left(E_{1}\right) t\right]=\operatorname{Proj}\left(\bigoplus_{n \geq 0} F_{e}\left(E_{1}\right)^{n} t^{n}\right) .
$$

Note that the surjection $\nu$ implies an inclusion of the respective Fitting ideals, and so we have

$$
[[E]]_{R} \simeq\left[\left[E_{1}\right]\right]_{R} \simeq F_{e}\left(E_{1}\right) \subseteq F_{e}(E) .
$$

Using (2.12) we obtain another inequality for the analytic spread of $\bigwedge^{e} E$.

Proposition 2.4. Let $R$ be a Noetherian local ring and $E$ a finitely generated $R$ module having rank $e>0$, but not free. Then, there exists a module $E_{1}$ of projective dimension 1 having rank e such that $E_{1} / \tau_{R}\left(E_{1}\right) \simeq E$, and

$$
\ell\left(\bigwedge^{e} E\right) \geq \text { ht } F_{e}\left(E_{1}\right) \text {. }
$$

Proof. Let $E_{1}$ constructed as above. Then

$$
\begin{aligned}
\ell\left(\bigwedge^{e} E\right) & =\ell\left(\bigwedge^{e} E / \tau_{R}\left(\bigwedge^{e} E\right)\right)=\ell\left(\operatorname{det}_{0}(E)\right)=\ell\left([[E]]_{R}\right) \\
& =\ell\left(F_{e}\left(E_{1}\right)\right) \geq \operatorname{ht} F_{e}\left(E_{1}\right),
\end{aligned}
$$

as asserted.

In particular, if projdim $E \leq 1$ then $E=E_{1}$, and we get another affirmative answer for the problem mentioned in [17], without additional assumptions on $R$.

Corollary 2.5. Let $R$ be a Noetherian local ring and $E$ a finitely generated $R$-module having rank e with proj $\operatorname{dim} E=1$. Then

$$
\ell\left(\bigwedge^{e} E\right) \geq \operatorname{ht} F_{e}(E) .
$$

The module $E_{1}$ is also useful to prove the equality of the first non-zero Fitting ideals of $E$ and $G / E$, for ideal modules having projective dimension equal to one. We note that this equality was proved in [6] in the case where $R$ is a 2-dimensional regular local ring.

Proposition 2.6. Let $R$ be a Noetherian ring and $E \subseteq G \simeq R^{e}$ a finitely generated torsionfree $R$-module having rank $e>0$. Then, up to isomorphism,

$$
F_{0}(G / E) \subseteq F_{e}(E) .
$$

Moreover, if $E$ is an ideal module with proj $\operatorname{dim} E=1$ then

$$
F_{0}(G / E)=F_{e}(E) \text {. }
$$


Proof. Let $E_{1}$ constructed as above. Using Eq. (2.5), (2.11) and (2.12) we get

$$
F_{0}(G / E)=\operatorname{det}_{0}(E) \simeq[[E]]_{R} \simeq F_{e}\left(E_{1}\right) \subseteq F_{e}(E) .
$$

Now, if proj $\operatorname{dim} E=1$ then $F_{e}\left(E_{1}\right)=F_{e}(E)$. Moreover, if $E$ is an ideal module then

$$
\text { grade } F_{0}(G / E)=\text { grade } F_{e}(E)=\text { grade } G / E \geq 2,
$$

and so $F_{0}(G / E)=F_{e}(E)$, as asserted.

2.5. The universal property of the blow up at a module. Next we show that the blow up at the order determinant $\operatorname{det}_{0}(E)$ has an universal flattening property. This gives an alternative to [22, Theorem 3.3].

To do this we begin to prove an easy consequence of Lipman's Theorem (see [9, Theorem D.18]).

Proposition 2.7. Let $R$ be a Noetherian local ring and $E \subseteq G \simeq R^{e}$ a finitely generated torsionfree $R$-module having rank $e>0$. Then the following are equivalent:

a) $E$ is free.

b) $F_{0}(G / E)$ is a principal ideal (generated by a non-zero divisor of $R$ ).

c) $\operatorname{det}_{0}(E)$ is invertible.

Proof. "(a) $\Rightarrow$ (b)" If $E$ is free, we have a natural exact sequence $0 \rightarrow E \rightarrow G \rightarrow$ $G / E \rightarrow 0$ with $E$ and $G$ free $R$-modules. Since $G / E$ has rank 0 then $\tau_{R}(G / E)=$ $G / E$ and so (b) follows by Lipman's Theorem.

"(b) $\Leftrightarrow(\mathrm{c})$ " is clear.

"(b) $\Rightarrow$ (a)" By Lipman's Theorem, proj $\operatorname{dim} G / E \leq 1$, and so proj $\operatorname{dim} E=0$ proving that $E$ is free.

Under our conditions, the ideal $\operatorname{det}_{0}(E)$ behaves well by localization as well as the computation of the inverse. Therefore the ideal $\operatorname{det}_{0}(E) \operatorname{det}_{0}(E)^{-1} \operatorname{defines~the~}^{-}$ non-free locus of $E$.

Corollary 2.8. Let $R$ be a Noetherian ring and $E \subseteq G \simeq R^{e}$ a finitely generated torsionfree $R$-module having rank $e>0$. Then

$$
V\left(\operatorname{det}_{0}(E) \operatorname{det}_{0}(E)^{-1}\right)=\operatorname{Spec}(R) \backslash V\left(F_{e}(E)\right) .
$$

Proof. Let $\mathfrak{p}$ be an $R$-prime ideal. We have

$$
\begin{aligned}
E_{\mathfrak{p}} \text { is free } & \Longleftrightarrow \operatorname{det}_{0}\left(E_{\mathfrak{p}}\right)=\operatorname{det}_{0}(E)_{\mathfrak{p}} \text { is invertible } \\
& \Longleftrightarrow\left(\operatorname{det}_{0}(E)_{\mathfrak{p}}\right)\left(\operatorname{det}_{0}(E)_{\mathfrak{p}}\right)^{-1}=R_{\mathfrak{p}} \\
& \Longleftrightarrow\left(\operatorname{det}_{0}(E) \operatorname{det}_{0}(E)^{-1}\right)_{\mathfrak{p}}=R_{\mathfrak{p}} \\
& \Longleftrightarrow \mathfrak{p} \nsupseteq\left(\operatorname{det}_{0}(E)\right)\left(\operatorname{det}_{0}(E)\right)^{-1}
\end{aligned}
$$

and the equality follows.

We say that a ring homomorphism $\varphi: R \rightarrow S$ is birational if $\varphi$ induces an isomorphim $\mathrm{Q}(R) \simeq \mathrm{Q}(S)$ between the total ring of fractions. 
Proposition 2.9. Let $R$ be a Noetherian ring and $E$ a finitely generated $R$-module having rank e. Let $\varphi: R \rightarrow S$ be a birational homomorphism of rings. Then

$$
E \otimes_{R} S / \tau_{R}\left(E \otimes_{R} S\right) \text { is free } \Longleftrightarrow \operatorname{det}_{0}(E) \cdot S \text { is invertible. }
$$

Proof. Since $E$ has rank $e$ there exists an $R$-homomorphism $f: E \rightarrow R^{e}$ with rank $f=e$. By Properties 2.1

$$
\operatorname{det}_{f \otimes \mathrm{id}}\left(E \otimes_{R} S / \tau_{R}\left(E \otimes_{R} S\right)\right)=\operatorname{det}_{f \otimes \mathrm{id}}\left(E \otimes_{R} S\right)=\operatorname{det}_{f}(E) \cdot S .
$$

Now we observe that $E \otimes_{R} S$ has rank $e$. In fact, since $\varphi$ is birational

$$
\begin{aligned}
\left(E \otimes_{R} S\right) \otimes_{S} \mathrm{Q}(S) & \simeq E \otimes_{R} \mathrm{Q}(S) \simeq E \otimes_{R} \mathrm{Q}(R) \otimes_{\mathrm{Q}(R)} \mathrm{Q}(S) \\
& \simeq \mathrm{Q}(R)^{e} \otimes_{\mathrm{Q}(R)} \mathrm{Q}(S) \simeq \mathrm{Q}(S)^{e} .
\end{aligned}
$$

Moreover, we also have

$$
\operatorname{im}(\overline{f \otimes i d}) \otimes_{S} \mathrm{Q}(S)=\left(\operatorname{im} f \otimes_{R} S\right) \otimes_{S} \mathrm{Q}(S) \simeq \mathrm{Q}(S)^{e} .
$$

Therefore $f \otimes$ id has rank $e$ and, by Lemma $2.2, \overline{f \otimes i d}$ is an embedding. Now the result follows by Proposition 2.7 .

Following the notation of [22], for a given scheme $\left(X, \mathcal{O}_{X}\right)$ we denote by $\overline{\mathrm{Q}(X)}$ the sheaf of total quotient rings of $\mathcal{O}_{X}$. Then, for a given sheaf $\mathcal{E}$ of $\mathcal{O}_{X}$-modules, we also denote by $\operatorname{tor}(\mathcal{E})$ the subsheaf of torsion of $\mathcal{E}$, so that $\mathcal{E} / \operatorname{tor}(\mathcal{E})$ is a sheaf of torsionfree $\mathcal{O}_{X}$-modules.

Theorem 2.10. Let $R$ be a reduced Noetherian ring, $E$ a finitely generated $R$-module having positive rank e and $\operatorname{det}_{0}(E)$ the order determinant of $E$. Let $X \stackrel{\pi}{\longrightarrow} \operatorname{Spec}(R)$ be the blow up of $R$ with center $\operatorname{det}_{0}(E)$. Then

a) $\pi^{*}(E) / \operatorname{tor}\left(\pi^{*}(E)\right)$ is a locally free sheaf of $\mathcal{O}_{X}$-modules of rank e.

b) For any birational morphism $Y \stackrel{\gamma}{\longrightarrow} \operatorname{Spec}(R)$ for which $\gamma^{*}(E) / \operatorname{tor}\left(\gamma^{*}(E)\right)$ is locally free of rank $e$, there is a unique morphism $\beta: Y \rightarrow X$ such that $\beta \circ \pi=\gamma$.

Proof. The proof is direct, just taking into account that under our conditions the blow up is a birational morphism, the universal property of the blow up, Proposition 2.9, and that we only need to check it locally.

\section{ReduCtion OF MOdules}

We briefly recall the definition of reduction of a module, and notions around, and establish some basic properties that we need in the next section.

Suppose that $E$ is a finitely generated $R$-module having a rank over a Noetherian ring $R$. We denote by $\mathcal{R}(E)$ the Rees algebra of $E$, which is by definition the symmetric algebra module its torsion $R$-submodule. An $R$-module $U$ of $E$ is said to be a reduction of $E$ if

$$
\mathcal{R}(E)_{r+1}=\left(U / \tau_{R}(U)\right) \cdot \mathcal{R}(E)_{r}
$$

for some $r \geq 0$. Since $E$ and $E / \tau_{R}(E)$ have the same rank and the same Rees algebra, then $U$ is a reduction of $E$ if and only if $U / \tau_{R}(U)$ is a reduction of $E / \tau_{R}(E)$. Hence, we may often assume that $E$ is torsionfree, since the theory of reductions for 
torsionfree modules affords some additional properties. The least integer $r$ for which $\mathcal{R}(E)_{r+1}=\left(U / \tau_{R}(U)\right) \cdot \mathcal{R}(E)_{r}$ is called the reduction number of $E$ with respect to $U$, and is denoted by $r_{U}(E)$.

The Rees algebra does not commute in general with the extension of scalars. A simple example was given by A. Micali in [11, Chap. I, $\S 5$, Example]: Let $(R, \mathfrak{m})$ be a local domain and let $k=R / \mathfrak{m}$. Then, $\mathcal{R}_{R}(k) \otimes_{R} k \simeq R \otimes_{R} k \simeq k$ while $\mathcal{R}_{k}\left(k \otimes_{R}\right.$ $k) \simeq k[t]$. Nevertheless, because the symmetric algebra always commutes with the extension of scalars, the Rees algebra commutes if the torsion also commutes. In particular, we have that the Rees algebra commutes with polynomial and Nagata extensions, which implies that reductions are preserved by polynomial and Nagata extensions.

Let $E$ be a finitely generated torsionfree $R$-module having rank. A reduction of $E$ is called minimal if it is minimal with respect to inclusion. Minimal reductions always exist. The reduction number of $E$, denoted by $r(E)$, is the minimum of $r_{U}(E)$, where $U$ ranges over all minimal reductions of $E$.

Over an integral domain the order determinants can be used to check whether a submodule can be a reduction of a module (cf. [21, Proposition 8.66]). Since, under the assumption that $\operatorname{proj} \operatorname{dim} E=1$, we have

$$
\operatorname{det}_{0}(E)=F_{e}(E) \text {, }
$$

then we may assert the following:

Proposition 3.1. Let $R$ be an integral domain and let $U$ and $E$ be finitely generated torsionfree $R$-modules of rank $e$ with $U \subset E$. Suppose that proj $\operatorname{dim} E=$ proj $\operatorname{dim} U=1$. Then $U$ is a reduction of $E$ if and only if $F_{e}(U)$ is a reduction of $F_{e}(E)$.

In [3, Proposition 2.1] or [13, Theorem 3.3] we can find the fundamental properties of reduction of modules. For completeness we prove that the minimal number of generators of a finitely generated torsionfree $R$-module $E$ and of any minimal reduction are well related as in the case of ideals.

Lemma 3.2. Let $(R, \mathfrak{m})$ be a Noetherian local ring, $E$ a finitely generated torsionfree $R$-module and $U$ an $R$-submodule of $E$. Then $U$ is a reduction of $E$ if and only if $U+\mathfrak{m} E$ is a reduction of $E$.

Proof. Suppose that $U$ is a reduction of $E$. Then $\mathcal{R}(E)_{r+1}=U \cdot \mathcal{R}(E)_{r}$ for some $r \geq 0$ and we have

$$
(U+\mathfrak{m} E) \cdot \mathcal{R}(E)_{r}=U \cdot \mathcal{R}(E)_{r}+(\mathfrak{m} E) \cdot \mathcal{R}(E)_{r}=\mathcal{R}(E)_{r+1} .
$$

Conversely, assume that $(U+\mathfrak{m} E) \cdot \mathcal{R}(E)_{r}=\mathcal{R}(E)_{r+1}$ for some $r \geq 0$. Then $\mathcal{R}(E)_{r+1}=(U+\mathfrak{m} E) \cdot \mathcal{R}(E)_{r}=U \cdot \mathcal{R}(E)_{r}+(\mathfrak{m} E) \cdot \mathcal{R}(E)_{r}=U \cdot \mathcal{R}(E)_{r}+\mathfrak{m} \mathcal{R}(E)_{r+1}$ and so, by Nakayama's lemma, $\mathcal{R}(E)_{r+1}=U \cdot \mathcal{R}(E)_{r}$.

Proposition 3.3. Let $(R, \mathfrak{m}, k)$ be a Noetherian local ring and $E$ a finitely generated torsionfree $R$-module having rank. If $U=R a_{1}+\cdots+R a_{n}$ is a minimal reduction of $E$ with $n=\mu(U)$, then $\bar{a}_{1}, \ldots, \bar{a}_{n}$ are linearly independent over $k$, where $\bar{a}_{i}=a_{i}+\mathfrak{m} E$. 
In particular $a_{1}, \ldots, a_{n}$ are part of a minimal system of generators of $E$. Moreover, $U \cap \mathfrak{m} E=\mathfrak{m} U$ and $\mu(E)=\mu(U)+\mu(E / U)$.

Proof. In fact, if $\bar{a}_{1}, \ldots, \bar{a}_{n}$ are not linearly independent over $k$, without loss of generality we may assume that $\bar{a}_{n} \in\left\langle\bar{a}_{1}, \ldots, \bar{a}_{n-1}\right\rangle$, so $V=R a_{1}+\cdots+R a_{n-1} \varsubsetneqq U$ is also a reduction of $E$ (by Lemma 3.2), contradicting the minimality of $U$. Hence $\bar{a}_{1}, \ldots, \bar{a}_{n}$ are linearly independent over $k$ and so $U \cap \mathfrak{m} E=\mathfrak{m} U$. This implies that any minimal generating set of $U$ can be extended to a minimal generating set of $E$. In particular, $a_{1}, \ldots, a_{n}$ are part of a minimal system of generators of $E$. Furthermore,

$$
0 \rightarrow U / \mathfrak{m} U \simeq(\mathfrak{m} E+U) / \mathfrak{m} E \rightarrow E / \mathfrak{m} E \rightarrow E /(\mathfrak{m} E+U) \simeq(E / U) / \mathfrak{m}(E / U) \rightarrow 0
$$

is an exact sequence. Hence

$$
\mu(E)=\operatorname{dim}_{k}(E / \mathfrak{m} E)=\operatorname{dim}_{k}(U / \mathfrak{m} U)+\operatorname{dim}_{k}((E / U) / \mathfrak{m}(E / U))=\mu(U)+\mu(E / U),
$$

and the result follows.

Corollary 3.4. Let $(R, \mathfrak{m})$ be a Noetherian local ring and $E$ a finitely generated $R$-module having positive rank. If $U$ is a reduction of $E$ then $U \nsubseteq \mathfrak{m} E$.

Proof. Since if $U \subseteq \mathfrak{m} E$ then $U / \tau_{R}(U) \subseteq \mathfrak{m}\left(E / \tau_{R}(E)\right)$, we may assume that $E$ is torsionfree. Let $V \subseteq U$ be a minimal reduction of $E$. We begin to prove that $V \nsubseteq \mathfrak{m} E$. If not, since $V \cap \mathfrak{m} E=\mathfrak{m} V$ (by Proposition 3.3) we deduce that $V=\mathfrak{m} V$. Hence, by Nakayama's Lemma, $V=0$, a contradiction. Therefore $V \nsubseteq \mathfrak{m} E$, and so $U \nsubseteq \mathfrak{m} E$.

Any reduction $U$ of $E$ has rank and $\operatorname{rank} U=\operatorname{rank} E$. Moreover, $E / U$ is a torsion module and grade $E / U>0$. Further, if $V \subset U$ with $E / V$ having rank then $U / V$ is a reduction of $E / V$ (see [4, Proposition 2.4]).

Given a Noetherian local ring $(R, \mathfrak{m}, k)$ and a finitely generated torsionfree $R$-module $E$ having rank, it is known that

$$
\mu(U) \geq \ell(E)=\operatorname{dim} \mathcal{F}(E)
$$

for any reduction $U$ of $E$ with equality if and only if $U$ is a minimal reduction of $E$, supposing $k$ infinite. In fact, in this case the classes in $E / \mathfrak{m} E$ of any minimal generating set of $U$ are a system of parameters of $\mathcal{F}(E)=\mathcal{R}(E) \otimes_{R} R / \mathfrak{m}$.

\section{The Quotient module $\bar{E}=E^{\prime \prime} / F$}

In this section we recall the construction of a generic Bourbaki ideal given in [18. We explore the inductive process of this construction in order to prove a formula for the minimal number of generators of a generic Bourbaki ideal. We shall do this more generally for a quotient module $\bar{E}$ of rank 1 (not necessarily an ideal). This result will be applied in the next section mainly in the case of modules with projective dimension one. 
Let $(R, \mathfrak{m})$ be a Noetherian local ring, and $E$ a finitely generated $R$-module having a rank $e>0$, and let $U=\sum_{i=1}^{n} R a_{i}$ be a submodule of $E$ such that $E / U$ is a torsion module (which holds if $U$ is a reduction of $E$ ). Further, let $\mathbf{Z}=\left\{z_{i j} \mid 1 \leq i \leq n, 1 \leq\right.$ $j \leq e-1\}$ be a set of $n \times(e-1)$ indeterminates over $R$. We fix the notation

$$
R^{\prime}=R\left[z_{i j} \mid 1 \leq i \leq n, 1 \leq j \leq e-1\right]=R[\mathbf{Z}], R^{\prime \prime}=R_{\mathfrak{m} R^{\prime}}^{\prime}=R(\mathbf{Z}) .
$$

The ring $R^{\prime \prime}$ is a local ring with maximal ideal $\mathfrak{m} R^{\prime \prime}$ and infinite residue field $k(\mathbf{Z})$ and is called the Nagata extension of $R$ with respect to $\mathbf{Z}$. Further, we set

$$
\begin{gathered}
U^{\prime}=U \otimes_{R} R^{\prime}, U^{\prime \prime}=U \otimes_{R} R^{\prime \prime}, E^{\prime}=E \otimes_{R} R^{\prime}, E^{\prime \prime}=E \otimes_{R} R^{\prime \prime}, \\
x_{j}=\sum_{i=1}^{n} z_{i j} a_{i} \in U^{\prime} \subseteq E^{\prime}, F=\sum_{j=1}^{e-1} R^{\prime \prime} x_{j} .
\end{gathered}
$$

In [18] it is proved that $F$ is a free module over $R^{\prime \prime}$ of rank $e-1$. We denote the $R^{\prime \prime}$-module $E^{\prime \prime} / F$ by $\bar{E}$; this module has rank 1 . There are good relations between some numerical invariants of $\bar{E}$ and $E$. In particular, the Krull dimensions of their Rees algebras and of their fiber cones are related by the equalities

$$
\begin{aligned}
& \operatorname{dim} \mathcal{R}(E)=\operatorname{dim} \mathcal{R}(\bar{E})+e-1, \\
& \ell(E)=\ell(\bar{E})+e-1 .
\end{aligned}
$$

In [4] we explored the inductive process of this construction and made deeper the relations between $E$ and $\bar{E}$. In particular, we proved that, under certain conditions, the generators $x_{1}, \ldots, x_{e-1}$ of $F$ form a regular sequence on $\mathcal{R}\left(E^{\prime \prime}\right)$ and

$$
\mathcal{R}(\bar{E}) \simeq \mathcal{R}\left(E^{\prime \prime}\right) /\left\langle x_{1}, \ldots, x_{e-1}\right\rangle
$$

(cf [4, Theorem 3.7]), hence $\mathcal{R}\left(E^{\prime \prime}\right)$ is a deformation of $\mathcal{R}(\bar{E})$. As a consequence we got

$$
\operatorname{depth} \mathcal{R}(E)=\operatorname{depth} \mathcal{R}(\bar{E})+e-1 .
$$

In this section, following our approach in [4], we prove that

$$
\mu(\bar{E})=\mu(E)+e-1 .
$$

This equality allow us to construct a minimal generating set $x_{1}, \ldots, x_{e-1}, \ldots, x_{n}$ of $E^{\prime \prime}$ containing the generators $x_{1}, \ldots, x_{e-1}$ of $F$.

We use the same notation as in [4]. For $1 \leq j \leq e-1$ set

$$
\mathbf{Z}_{j}=\left\{z_{1 j}, \ldots, z_{n j}\right\}
$$

and

$$
R_{j}=R\left[\mathbf{Z}_{1}, \ldots, \mathbf{Z}_{j}\right], R_{j}^{\prime \prime}=R_{j_{\mathfrak{m} R_{j}}}=R\left(\mathbf{Z}_{1}, \ldots, \mathbf{Z}_{j}\right)
$$

Then

$$
R_{j}=R_{j-1} \otimes_{R} R\left[\mathbf{Z}_{j}\right]=R_{j-1}\left[\mathbf{Z}_{j}\right] .
$$

According to [4, Lemma 3.1], we have

$$
\begin{gathered}
R^{\prime} \simeq R_{j} \otimes_{R} R\left[\mathbf{Z}_{j+1}, \ldots, \mathbf{Z}_{e-1}\right] \simeq R_{j}\left[\mathbf{Z}_{j+1}, \ldots, \mathbf{Z}_{e-1}\right] \\
R^{\prime \prime} \simeq\left(R_{j}^{\prime \prime}\left[\mathbf{Z}_{j+1}, \ldots, \mathbf{Z}_{e-1}\right]\right)_{\mathfrak{m} R_{j}^{\prime \prime}\left[\mathbf{Z}_{j+1}, \ldots, \mathbf{Z}_{e-1}\right]}=R_{j}^{\prime \prime}\left(\mathbf{Z}_{j+1}, \ldots, \mathbf{Z}_{e-1}\right),
\end{gathered}
$$


and

$$
\begin{aligned}
R_{j}^{\prime \prime} & \simeq\left(R_{j-1}^{\prime \prime}\left[\mathbf{Z}_{j}\right]\right)_{\mathfrak{m} R_{j-1}^{\prime \prime}\left[\mathbf{Z}_{j}\right]}=R_{j-1}^{\prime \prime}\left(\mathbf{Z}_{j}\right)=R\left(\mathbf{Z}_{1}, \ldots, \mathbf{Z}_{j-1}\right)\left(\mathbf{Z}_{j}\right) \\
& \simeq R\left(\mathbf{Z}_{j}\right)\left(\mathbf{Z}_{1}, \ldots, \mathbf{Z}_{j-1}\right)=\left(\left(R\left[\mathbf{Z}_{j}\right]_{\mathfrak{m} R\left[\mathbf{Z}_{j}\right]}\right)\left[\mathbf{Z}_{1}, \ldots, \mathbf{Z}_{j-1}\right]\right)_{\mathfrak{m}\left(R\left[\mathbf{Z}_{j}\right]_{\mathfrak{m} R\left[\mathbf{Z}_{j}\right]}\right]\left[\mathbf{Z}_{1}, \ldots, \mathbf{Z}_{j-1}\right]}
\end{aligned}
$$

Moreover, for $1 \leq j \leq e-1$, set $E_{j}=E \otimes_{R} R_{j}, E_{j}^{\prime \prime}=E \otimes_{R} R_{j}^{\prime \prime} \simeq E_{j} \otimes_{R_{j}} R_{j}^{\prime \prime}$. Hence

$$
\begin{gathered}
E_{j}=E_{j-1} \otimes_{R} R\left[\mathbf{Z}_{j}\right]=E_{j-1} \otimes_{R_{j-1}} R_{j}, \\
E^{\prime} \simeq E_{j} \otimes_{R_{j}} R^{\prime} \simeq E_{j} \otimes_{R} R\left[\mathbf{Z}_{j+1}, \ldots, \mathbf{Z}_{e-1}\right], \\
E^{\prime \prime} \simeq E^{\prime} \otimes_{R^{\prime}} R^{\prime \prime} \simeq E_{j} \otimes_{R_{j}} R^{\prime \prime} \simeq E_{j}^{\prime \prime} \otimes_{R_{j}^{\prime \prime}} R^{\prime \prime}, \\
E_{j}^{\prime \prime} \simeq E_{j-1}^{\prime \prime} \otimes_{R_{j-1}^{\prime \prime}} R_{j}^{\prime \prime}, \overline{E_{j}^{\prime \prime}} \simeq \overline{E_{j}} \otimes_{R_{j}} R_{j}^{\prime \prime} .
\end{gathered}
$$

Furthermore, for $1 \leq j \leq e-1$, set $\overline{E_{j}}=E_{j} /\left\langle x_{1}, \ldots, x_{j}\right\rangle, \overline{E_{j}^{\prime \prime}}=E_{j}^{\prime \prime} /\left\langle x_{1}, \ldots, x_{j}\right\rangle$, where $\left\langle x_{1}, \ldots, x_{j}\right\rangle$ denote in each case the submodule generated by $x_{1}, \ldots, x_{j}$. By convention $\overline{E_{0}}=E=E_{0}, R_{0}=R$. We have, for each $1 \leq j \leq e-1$,

$$
\overline{E_{j}} \simeq\left(\overline{E_{j-1}} \otimes_{R_{j-1}} R_{j}\right) /\left\langle\overline{x_{j}}\right\rangle, \overline{E_{j}^{\prime \prime}} \simeq\left(\overline{E_{j-1}^{\prime \prime}} \otimes_{R_{j-1}^{\prime \prime}} R_{j}^{\prime \prime}\right) /\left\langle\overline{x_{j}}\right\rangle,
$$

where $\overline{x_{j}}=x_{j}+\left\langle x_{1}, \ldots, x_{j-1}\right\rangle$ in each case (cf. [4, Lemma 3.2]). These relations for $E$ are also true for any submodule $U$ of $E$. Moreover, since $R^{\prime \prime}$ is the Nagata extension of $R_{j}^{\prime \prime}$ with respect to $\mathbf{Z}_{j+1}, \ldots, \mathbf{Z}_{e-1}$,

$$
\operatorname{rank} \overline{E_{j}^{\prime \prime}}=\operatorname{rank} \overline{U_{j}^{\prime \prime}}=e-j \geq 2, \mu\left(E_{j}^{\prime \prime}\right)=\mu\left(E^{\prime \prime}\right)
$$

(cf. [4, Lemma 3.3]).

For the module $\bar{E}$ constructed above we are able to prove that $\mu(\bar{E})=\mu(E)-e+1$. We argue by induction on the rank of $F$.

Lemma 4.1. Let $(R, \mathfrak{m}, k)$ be a Noetherian local ring, $E$ a finitely generated $R$-module having rank $e \geq 2$ and $U=\sum_{i=1}^{n} R a_{i}$ a reduction of $E$. Let $R^{\prime}=R\left[z_{1}, \ldots, z_{n}\right]$, $R^{\prime \prime}=R_{\mathfrak{m} R^{\prime}}^{\prime}, x=z_{1} a_{1}+\cdots+z_{n} a_{n}$ and let $U^{\prime \prime}=U \otimes_{R} R^{\prime \prime}, E^{\prime \prime}=E \otimes_{R} R^{\prime \prime}$. Then $\mu\left(V^{\prime \prime} / R^{\prime \prime} x\right)=\mu(V)-1$, for every $R$-submodule $V$ of $E$ containing $U$ and $V^{\prime \prime}=V \otimes_{R} R^{\prime \prime}$.

Proof. Let $V$ be any $R$-module such that $U \subseteq V \subseteq E$. Since $U=\sum_{i=1}^{n} R a_{i} \nsubseteq \nsubseteq \mathfrak{m} E$ (by Corollary 3.4) there exists an $i$ such that $a_{i} \notin \mathfrak{m} E$. Without loss of generality we may assume that $a_{1} \notin \mathfrak{m} E$. We claim that $x / 1 \in U^{\prime \prime} \subseteq V^{\prime \prime} \subseteq E^{\prime \prime}$ is part of a minimal generating set of $V^{\prime \prime}$. Suppose not, that is $x / 1 \in \mathfrak{m} V^{\prime \prime}$. Since $V^{\prime \prime}=V^{\prime} \otimes_{R^{\prime}} R_{\mathfrak{m} R^{\prime}}^{\prime}=V_{\mathfrak{m} R^{\prime}}^{\prime}$ there exists $y \in R^{\prime} \backslash \mathfrak{m} R^{\prime}$ such that $y x \in \mathfrak{m} V^{\prime}$. Set

$$
\bar{y}=y+\mathfrak{m} R^{\prime} \in R^{\prime} / \mathfrak{m} R^{\prime} \simeq R / \mathfrak{m} \otimes_{R} R^{\prime} \simeq k\left[z_{1}, \ldots, z_{n}\right],
$$

and set

$$
\bar{x}=x+\mathfrak{m} V^{\prime}=\overline{a_{1}} z_{1}+\cdots+\overline{a_{n}} z_{n} \in V^{\prime} / \mathfrak{m} V^{\prime}=V / \mathfrak{m} V \otimes_{R} R^{\prime}=(V / \mathfrak{m} V)\left[z_{1}, \ldots, z_{n}\right],
$$

with $\overline{a_{i}}=a_{i}+\mathfrak{m} V \in V / \mathfrak{m} V,(i=1, \ldots, n)$. Hence $\bar{y} \bar{x}=0$ with $\bar{y} \neq 0$. Now write $\bar{y}=\sum_{j=0}^{m} \overline{y_{j}} z_{1}^{j}$ with $\overline{y_{j}} \in k\left[z_{2}, \ldots, z_{n}\right],(j=0, \ldots, m)$ and $\overline{y_{m}} \neq 0$. Hence, we have

$$
\bar{y} \bar{x}=\sum_{i=1}^{n} \bar{y} \overline{a_{i}} z_{i}=\bar{y} \overline{a_{1}} z_{1}+\sum_{i=2}^{n} \bar{y} \overline{a_{i}} z_{i}=\sum_{j=0}^{m} \overline{y_{j}} \overline{a_{1}} z_{1}^{j+1}+\sum_{i=2}^{n} \bar{y} \overline{a_{i}} z_{i}
$$


and since $\bar{y} \bar{x}=0$ we must have $\overline{y_{m}} \overline{a_{1}} z_{1}^{m+1}=0$. Hence $\overline{y_{m}} \overline{a_{1}}=0$. But $\overline{y_{m}} \overline{a_{1}} \in$ $V / \mathfrak{m} V\left[z_{2}, \ldots, z_{n}\right]=k\left[z_{2}, \ldots, z_{n}\right] \otimes_{k} V / \mathfrak{m} V$ with $k\left[z_{2}, \ldots, z_{n}\right]$ faithfully flat over $k$ and $0 \neq \overline{y_{m}} \in k\left[z_{2}, \ldots, z_{n}\right], \overline{a_{1}}=a_{i}+\mathfrak{m} V \neq 0$ (because $a_{1} \notin \mathfrak{m} V \subseteq \mathfrak{m} E$ ). Therefore $x / 1 \notin \mathfrak{m} V^{\prime \prime}$ and $x / 1$ is part of a minimal generating set of $V^{\prime \prime}$, as claimed. It follows that

$$
\mu\left(V^{\prime \prime} / R^{\prime \prime} x\right)=\mu\left(V^{\prime \prime}\right)-1=\mu(V)-1,
$$

proving the assertion.

Lemma 4.2. Let $(R, \mathfrak{m}, k)$ be a Noetherian local ring, let $E$ be a finitely generated $R$-module having rank $e \geq 2$ and $U=\sum_{i=1}^{n} R a_{i}$ a reduction of $E$. Let $R^{\prime \prime}$, $x_{1}, \ldots, x_{e-1}, F$ be defined as before. Set, for each $1 \leq t \leq e-1, F_{t}=\sum_{i=1}^{t} R^{\prime \prime} x_{i}$. Then

a) $\mu\left(V^{\prime \prime} / F_{t}\right)=\mu(V)-t$,

b) $F_{t} \cap \mathfrak{m} R^{\prime \prime} V^{\prime \prime}=\mathfrak{m} R^{\prime \prime} F_{t}$,

for every $R$-submodule $V$ of $E$ containing $U, V^{\prime \prime}=V \otimes_{R} R^{\prime \prime}$.

Proof. Let $V$ be any $R$-module such that $U \subseteq V \subseteq E$ and set $V^{\prime \prime}=V \otimes_{R} R^{\prime \prime}$.

a) We use induction on $t$. For $t=1$ we apply the previous lemma, since $R_{1}=$ $R\left[z_{11}, \ldots, z_{n 1}\right]$ and $F_{1}$ is freely generated by $x_{1}=z_{11} a_{1}+\cdots+z_{n 1} a_{n}$. Suppose that $1 \leq j \leq t \leq e-1$. We set, as before, $E_{j}=E \otimes_{R} R_{j}, U_{j}=U \otimes_{R} R_{j}$ with $R_{j}=R\left[z_{11}, \ldots, z_{1 n}, \ldots, z_{1 j}, \ldots, z_{n j}\right]=R_{j-1}\left[\mathbf{Z}_{j}\right], R_{j}^{\prime \prime}=R_{j_{\mathfrak{m} R}}$ and $E_{j}^{\prime \prime}=E \otimes_{R} R_{j}^{\prime \prime}$, $U_{j}^{\prime \prime}=U \otimes_{R} R_{j}^{\prime \prime}$. Moreover, set $V_{j}=V \otimes_{R} R_{j}, V_{j}^{\prime \prime}=V \otimes_{R} R_{j}^{\prime \prime}=V_{j-1}^{\prime \prime} \otimes_{R_{j-1}^{\prime \prime}} R_{j}^{\prime \prime}$ and $F_{j}^{\prime \prime}=\sum_{i=1}^{j} R_{j}^{\prime \prime} x_{i}$. Since $R_{j}^{\prime \prime}$ is faithfully flat over $R, F_{j}^{\prime \prime} \subseteq U_{j}^{\prime \prime} \subseteq V_{j}^{\prime \prime} \subseteq E_{j}^{\prime \prime}$. Moreover, $F_{j}=F_{j}^{\prime \prime} \otimes_{R_{j}^{\prime \prime}} R^{\prime \prime}$ and $V^{\prime \prime}=\left(V \otimes_{R} R_{j}^{\prime \prime}\right) \otimes_{R_{j}^{\prime \prime}} R^{\prime \prime}=V_{j}^{\prime \prime} \otimes_{R_{j}^{\prime \prime}} R^{\prime \prime}$. Therefore

$$
V^{\prime \prime} / F_{j} \simeq V_{j}^{\prime \prime} / F_{j}^{\prime \prime} \otimes_{R_{j}^{\prime \prime}} R^{\prime \prime}
$$

Now, suppose for induction that $j>1$ and that $\mu\left(V^{\prime \prime} / F_{j-1}\right)=\mu(V)-(j-1)$. Hence, by Eq. (4.8),

$$
\mu\left(V_{j-1}^{\prime \prime} / F_{j-1}^{\prime \prime}\right)=\mu\left(V^{\prime \prime} / F_{j-1}\right)=\mu(V)-(j-1)=\mu\left(V_{j-1}^{\prime \prime}\right)-(j-1) .
$$

Moreover, $R_{j}^{\prime \prime}=R_{j-1}^{\prime \prime}\left(\mathbf{Z}_{j}\right)$ is the Nagata extension of $R_{j-1}^{\prime \prime}$ (with respect to $\mathbf{Z}_{j}$ ) and, as in Eq. (4.6), we have

$$
V_{j}^{\prime \prime} / F_{j}^{\prime \prime} \simeq\left(V_{j-1}^{\prime \prime} / F_{j-1}^{\prime \prime} \otimes_{R_{j-1}^{\prime \prime}} R_{j}^{\prime \prime}\right) /\left\langle\overline{x_{j}}\right\rangle
$$

where $\overline{x_{j}}=x_{j}+R_{j}^{\prime \prime} x_{1}+\cdots+R_{j}^{\prime \prime} x_{j-1}$. Further, $U_{j}^{\prime \prime} / F_{j}^{\prime \prime} \subseteq V_{j}^{\prime \prime} / F_{j}^{\prime \prime}$ with $U_{j}^{\prime \prime} / F_{j}^{\prime \prime}$ a reduction of $E_{j}^{\prime \prime} / F_{j}^{\prime \prime}$ and $\operatorname{rank}\left(E_{j}^{\prime \prime} / F_{j}^{\prime \prime}\right)=e-j \geq 2($ by Eq. (4.77)). Hence, by the previous lemma and by induction assumption,

$$
\mu\left(V_{j}^{\prime \prime} / F_{j}^{\prime \prime}\right)=\mu\left(V_{j-1}^{\prime \prime} / F_{j-1}^{\prime \prime}\right)-1=\mu\left(V_{j-1}^{\prime \prime}\right)-(j-1)-1=\mu\left(V_{j-1}^{\prime \prime}\right)-j .
$$

It follows by induction that

$$
\mu\left(V^{\prime \prime} / F_{t}\right)=\mu\left(V_{t}^{\prime \prime} / F_{t}^{\prime \prime}\right)=\mu\left(V_{t-1}^{\prime \prime}\right)-t=\mu(V)-t
$$

(since $R^{\prime \prime}$ is faithfully flat over $R_{j}^{\prime \prime}$ for all $j$ ), proving a).

b) is a direct consequence of a). 
Proposition 4.3. Let $R$ be a Noetherian local ring, let $E$ be a finitely generated $R$-module having rank $e \geq 2$ and $U$ a reduction of $E$. Let $R^{\prime \prime}, E^{\prime \prime}, x_{1}, \ldots, x_{e-1}, F$ be defined as before. Set, for each $1 \leq t \leq e-1, F_{t}=\sum_{i=1}^{t} R^{\prime \prime} x_{i}$. Then

a) $\mu\left(E^{\prime \prime} / F_{t}\right)=\mu(E)-t$;

b) $E^{\prime \prime} / F_{t}$ is a free $R^{\prime \prime}$-module if and only if $E$ is a free $R$-module.

Proof. a) is a particular case of the previous lemma.

b) Consider the natural exact sequence $0 \rightarrow F_{t} \rightarrow E^{\prime \prime} \rightarrow E^{\prime \prime} / F_{t} \rightarrow 0$.

If $E^{\prime \prime} / F_{t}$ is free, then $E^{\prime \prime} \simeq F_{t} \oplus E^{\prime \prime} / F_{t}$ and $E^{\prime \prime}$ is free over $R^{\prime \prime}$. Hence $E$ is free over $R$. Conversely, suppose that $E$ is free. Hence $\mu(E)=\operatorname{rank} E=e$. Thus, by a),

$$
\mu\left(E^{\prime \prime} / F_{t}\right)=\mu(E)-t=e-t=\operatorname{rank} E^{\prime \prime} / F_{t}
$$

and $E^{\prime \prime} / F_{t}$ is free.

Theorem 4.4. Let $(R, \mathfrak{m}, k)$ be a Noetherian local ring, let $E$ be a finitely generated $R$-module having rank $e \geq 2$ and $U$ a reduction of $E$. Let $\bar{E}=E^{\prime \prime} / F, \bar{U}=U^{\prime \prime} / F$ with $F \subset U^{\prime \prime} \subseteq E^{\prime \prime}$ as before. Then

a) $\mu(\bar{E})=\mu(E)-e+1$;

b) $\bar{E}$ is a free $R^{\prime \prime}$-module if and only if $E$ is a free $R$-module;

c) $\bar{E}$ has finite projective dimension if and only if $E$ has finite projective dimension; if this is the case, proj $\operatorname{dim} \bar{E}=\operatorname{proj} \operatorname{dim} E$;

d) Assume in addition that $E$ is torsionfree and $k$ is infinite. If $U$ is a minimal reduction of $E$ then $\bar{U}$ is a minimal reduction of $\bar{E}$.

Proof. a) and b) are particular cases of the previous result.

c) Consider the exact sequence $0 \rightarrow F \rightarrow E^{\prime \prime} \rightarrow \bar{E} \rightarrow 0$. Since $F$ is a free $R^{\prime \prime}$-module proj $\operatorname{dim} F=0$ and so the first assertion follows. The second is a consequence of $\mathrm{b}$ ) and of [8, Proposition VII.1.8].

d) We already observed that $\bar{U}$ is a reduction of $\bar{E}$. Now, we have

$$
\mu(\bar{U})=\mu(U)-e+1=\ell(E)-e+1=\ell(\bar{E}),
$$

(by Lemma 4.2, Eq. (3.2) and (4.2)), and so $\bar{U}$ is a minimal reduction of $\bar{E}$.

\section{Generic Bourbaki ideals and Fitting ideals}

Given a finitely generated torsionfree $R$-module $E$ having rank $e>0$ and satisfying $\widetilde{G_{2}}$ (that is $\mu\left(E_{\mathfrak{p}}\right) \leq e$ for all $\mathfrak{p}$ with depth $R_{\mathfrak{p}}=1$ ), and given a submodule $U$ of $E$ such that grade $E / U \geq 2$, there exists a Nagata extension $R^{\prime \prime}=R(\mathbf{Z})$ and a free $R^{\prime \prime}$-module $F \simeq R^{\prime \prime e-1}$ such that $\bar{E}=E^{\prime \prime} / F$ is torsionfree over $R^{\prime \prime}$ and having rank 1. In this case $\bar{E} \simeq I_{U}(E)$ is an $R^{\prime \prime}$-ideal (see [18, Proposition 3.2]). Such ideal $I=I_{U}(E)$ is called a generic Bourbaki ideal of $E$ with respect to $U$. If $U=E$ we simply write $I=I(E)$. The construction of a generic Bourbaki ideal depends on the generating set considered for $U$ and on the set of variables, but is essentially unique. See [18, Remark 3.4]. In particular, if $U$ is a reduction of $E$ and grade $F_{e}(E) \geq 2$ then there exists $I$ a generic Bourbaki ideal of $E$ with respect to $U$ with grade $I>0$ (see [4, Remark 4.1]). 
The aim of this section is to relate generic Bourbaki ideals with Fitting ideals. To do this we first observe that Fitting ideals are related, in a natural way, with the exterior algebra. Moreover, we observe that whenever we consider a finite presentation $\varphi$ for $E^{\prime \prime}$, with respect to a generating set containing a basis of $F$ there is a submatrix $\psi$ of $\varphi$ with the first non-zero Fitting ideal having grade $\geq 1$. This Fitting ideal is isomorphic to a generic Bourbaki ideal of $E$, and we use it to obtain information about the perfection of the ideal $I(E) \simeq E^{\prime \prime} / F$ (cf. Theorem 5.3). This is the main result of this section.

For any linear map $\Psi: R^{m} \rightarrow R^{n}$ the Fitting ideal $F_{n-m}\left(R^{n} / \operatorname{im} \Psi\right)=I_{m}(\Psi)$ can be obtained as a image of a given linear map $\bigwedge^{n-m} R^{n} \rightarrow \bigwedge^{n} R^{n} \simeq R$. Using the notation introduced in section 2 (in particular Eq. (2.3) ), we first note that for complementary subsets $H, K \subseteq[n]$, we have

$$
x_{H} \wedge x_{K}=(-1)^{\varepsilon_{H, K}} x_{1} \wedge x_{2} \wedge \cdots \wedge x_{n},
$$

where $\varepsilon_{H, K}$ is the number of ordered pairs $(i, j) \in H \times K$ such that $i>j$.

Lemma 5.1. Let $R$ be Noetherian ring and $\Psi: R^{m} \rightarrow R^{n}$ a linear map with $n \geq m$. Let $u_{1}, \ldots, u_{m} \in R^{n}$ be defined by the columns of $\Psi$ and consider the map

$$
\begin{aligned}
\theta: \bigwedge^{n-m} R^{n} & \longrightarrow \bigwedge^{n} R^{n} \\
x & \longmapsto x \wedge u_{1} \wedge \cdots \wedge u_{m} .
\end{aligned}
$$

Then $\operatorname{im} \theta=I_{m}(\Psi)$.

Proof. Suppose that $\Psi=\left(\alpha_{i j}\right)$ with respect to a basis $\left(v_{1}, \ldots, v_{m}\right)$ of $R^{m}$ and a basis $\left(e_{1}, \ldots, e_{n}\right)$ of $R^{n}$. Hence $\left(e_{H}\right)_{H \in \mathcal{P}_{n-m}([n])}$ is a basis of $\bigwedge^{n-m} R^{n}$. Moreover, since $u_{j}=\sum_{i=1}^{n} \alpha_{i j} e_{i}(j \in[m])$ then by Eq. (2.4),

$$
u_{1} \wedge \cdots \wedge u_{m}=\sum_{K \in \mathcal{P}_{m}([n])} \operatorname{det} \Psi_{K, J} e_{K}
$$

where $\Psi_{K, J}=\Psi\left[i_{1}, \ldots, i_{m} \mid 1, \ldots, m\right]$ for $K=\left\{i_{1}<\cdots<i_{m}\right\} \subset[n], J=[m]$. Therefore, for $H \in \mathcal{P}_{n-m}([n])$,

$$
\begin{aligned}
\theta\left(e_{H}\right) & =e_{H} \wedge \sum_{K \in \mathcal{P}_{m}([n])} \operatorname{det} \Psi_{K, J} e_{K} \\
& =\sum_{K \in \mathcal{P}_{m}([n])} \operatorname{det} \Psi_{K, J}\left(e_{H} \wedge e_{K}\right) \\
& \left.=\sum_{\substack{K \in \mathcal{P}_{m}([n]) \\
K \cap H=\emptyset}} \operatorname{det} \Psi_{K, J}\left(e_{H} \wedge e_{K}\right) \quad \quad \text { (since } z \wedge z=0, \forall z \in R^{n}\right) \\
& =\operatorname{det} \Psi_{[n] \backslash H, J}\left(e_{H} \wedge e_{[n] \backslash H}\right) \\
& =(-1)^{\varepsilon_{[n] \backslash H, J}} \operatorname{det} \Psi_{[n] \backslash H, J}\left(e_{1} \wedge \cdots \wedge e_{n}\right) .
\end{aligned}
$$

Hence $\theta$ is a matrix with one row and $\left(\begin{array}{l}n \\ m\end{array}\right)$ columns. Therefore,

$$
\operatorname{im} \theta=\left\langle\operatorname{det} \Psi_{[n] \backslash H, J}\right\rangle_{H \in \mathcal{P}_{n-m}([n])}=I_{m}(\Psi),
$$

as required. 
In the notation of section 4, suppose that $(R, \mathfrak{m})$ is a Noetherian local ring and $E$ is a finitely generated $R$-module having rank $e \geq 2$. Suppose also that $U=\sum_{i=1}^{k} R a_{i}$ is a reduction of $E$. Further set $R^{\prime}=R\left[z_{i j} \mid 1 \leq i \leq k, 1 \leq j \leq e-1\right]=R[\mathbf{Z}]$, $R^{\prime \prime}=R_{\mathfrak{m} R^{\prime}}^{\prime}=R(\mathbf{Z})$ and $E^{\prime \prime}=E \otimes_{R} R^{\prime \prime}$. Furthermore set

$$
x_{j}=\sum_{i=1}^{k} z_{i j} a_{i}, F=\sum_{j=1}^{e-1} R^{\prime \prime} x_{j}, \bar{E}=E^{\prime \prime} / F .
$$

We may extend the basis $\left(x_{1}, \ldots, x_{e-1}\right)$ of $F$ to a generating set $\left\{x_{1}, \ldots, x_{n}\right\}$ of $E^{\prime \prime}$. We note that, if necessary, we may choose it to be a minimal generating set of $E^{\prime \prime}$ since $\mu\left(E^{\prime \prime} / F\right)=\mu\left(E^{\prime \prime}\right)-e+1$ (by Theorem 4.4). Let

$$
R^{\prime \prime m} \stackrel{\varphi}{\rightarrow} R^{\prime \prime n} \stackrel{h}{\rightarrow} E^{\prime \prime} \rightarrow 0
$$

be a finite free presentation of $E^{\prime \prime}$ with respect to the generators $x_{1}, \ldots, x_{n}$, and write also by $\varphi$ the respective relation matrix. We have

$$
\left[x_{1} \cdots x_{n}\right] \varphi=0
$$

and

$$
\operatorname{rank} \varphi:=\operatorname{rank} \operatorname{im} \varphi=\operatorname{rank} K=n-e,
$$

where $K=\operatorname{ker} h$ is the module of relations of the generating set $\left\{x_{1}, \ldots, x_{n}\right\}$. Hence grade $I_{n-e}(\varphi) \geq 1$ and $I_{n-e+1}(\varphi)=0$ (by [2, Proposition 1.4.11]). Moreover, the matrix $\varphi$ has a particular form.

Lemma 5.2. In the above conditions, each row $i \in\{1, \ldots, e-1\}$ of $\varphi$ is a $Q$ linear combination of the rows $e, \ldots, n$, where $Q=\operatorname{Quot}\left(R^{\prime \prime}\right)$. In particular, $\varphi$ is $Q$-equivalent to $\left[\frac{0}{\Psi}\right]$, where $\Psi=\varphi[e, \ldots, n \mid 1, \ldots, m]$ is a submatrix of $\varphi$ satisfying grade $I_{n-e}(\Psi) \geq 1$.

Proof. The exact sequence $0 \rightarrow F \rightarrow E^{\prime \prime} \rightarrow \bar{E} \rightarrow 0$ yields the exact sequence

$$
0 \rightarrow F \otimes_{R^{\prime \prime}} Q \rightarrow E^{\prime \prime} \otimes_{R^{\prime \prime}} Q \rightarrow \bar{E} \otimes_{R^{\prime \prime}} Q \rightarrow 0 .
$$

Since $\bar{E} \otimes_{R^{\prime \prime}} Q$ is free

$$
E^{\prime \prime} \otimes_{R^{\prime \prime}} Q \simeq\left(F \otimes_{R^{\prime \prime}} Q\right) \oplus\left(\bar{E} \otimes_{R^{\prime \prime}} Q\right) .
$$

On the other hand, $\bar{\varphi}=\varphi \otimes_{R^{\prime \prime}}$ id presents $E^{\prime \prime} \otimes_{R^{\prime \prime}} Q$ with respect to the generators $x_{1} \otimes 1, \ldots, x_{n} \otimes 1$ and we have the exact sequence

$$
Q^{m} \stackrel{\bar{\varphi}}{\rightarrow} Q^{n} \rightarrow\left(F \otimes_{R^{\prime \prime}} Q\right) \oplus\left(\bar{E} \otimes_{R^{\prime \prime}} Q\right) \rightarrow 0 .
$$

Moreover, $\left(x_{1} \otimes 1, \ldots, x_{e-1} \otimes 1\right)$ is a basis of $F \otimes_{R^{\prime \prime}} Q$. Now, let $(v)$ be a basis of $\bar{E} \otimes_{R^{\prime \prime}} Q \simeq Q$ and let $\beta_{e}, \ldots, \beta_{n}$ be elements in $Q$ such that, for all $i \geq e$,

$$
x_{i} \otimes 1=\sum_{j=1}^{e-1} \gamma_{i j}\left(x_{j} \otimes 1\right)+\beta_{i} v,
$$


for some $\gamma_{i j} \in Q$. Let $\left(e_{1}, \ldots, e_{n}\right)$ be the canonical basis of $Q^{n}$. Let $\bar{h}=h \otimes$ id and suppose that $\varphi=\left(\alpha_{i j}\right)_{i, j}$. Since $\bar{h} \bar{\varphi}=0$ then, for all $1 \leq k \leq m$,

$$
\begin{aligned}
0 & =\sum_{i=1}^{e-1} \alpha_{i k}\left(x_{i} \otimes 1\right)+\sum_{i=e}^{n} \alpha_{i k}\left(x_{i} \otimes 1\right) \\
& =\sum_{i=1}^{e-1} \alpha_{i k}\left(x_{i} \otimes 1\right)+\sum_{i=e}^{n} \alpha_{i k}\left(\sum_{j=1}^{e-1} \gamma_{i j}\left(x_{j} \otimes 1\right)+\beta_{i} v\right) \\
& =\sum_{i=1}^{e-1} \alpha_{i k}\left(x_{i} \otimes 1\right)+\sum_{i=1}^{e-1}\left(\sum_{j=e}^{n} \alpha_{j k} \gamma_{j i}\right)\left(x_{i} \otimes 1\right)+\left(\sum_{i=e}^{n} \alpha_{i k} \beta_{i}\right) v \\
& =\sum_{i=1}^{e-1}\left(\alpha_{i k}+\sum_{j=e}^{n} \alpha_{j k} \gamma_{j i}\right)\left(x_{i} \otimes 1\right)+\left(\sum_{i=e}^{n} \alpha_{i k} \beta_{i}\right) v .
\end{aligned}
$$

Therefore $\sum_{i=e}^{n} \alpha_{i k} \beta_{i}=0$ and each row $i$ of $\varphi$, with $1 \leq i \leq e-1$, is a $Q$-linear combination of the rows $e, \ldots, n$. That is

$$
\varphi=\left[\begin{array}{c|c}
I_{e-1} & -\Gamma^{T} \\
\hline 0 & I_{n-e+1}
\end{array}\right]\left[\begin{array}{c}
0 \\
\hline \Psi
\end{array}\right]
$$

where $\Gamma=\left(\gamma_{k \ell}\right)$ is a $Q$-matrix of type $(e-1) \times(n-e+1)$ and $\Psi=\varphi[e, \ldots, n \mid 1, \ldots, m]$. Moreover, $I_{n-e}(\Psi) \cdot Q=I_{n-e}(\varphi) \cdot Q=Q$, and so grade $I_{n-e}(\Psi) \geq 1$, as claimed.

Let $\psi$ be an $(n-e+1) \times(n-e)$ submatrix of $\varphi$ satisfying grade $I_{n-e}(\psi) \geq 1$ (exists by Lemma 5.2). Since $\operatorname{rank} \operatorname{im} \psi=n-e$ (again by [2, Proposition 1.4.11]), rank ker $\psi=0$. But $\operatorname{ker} \psi$ is torsionfree, hence $\operatorname{ker} \psi=0$ and $\psi$ represents an injective linear map

$$
0 \rightarrow R^{\prime \prime n-e} \stackrel{\psi}{\rightarrow} R^{\prime \prime n-e+1}
$$

with respect to the canonical bases. Moreover, using Eq. (5.2), we get for any $j=1, \ldots, m$

$$
\sum_{i=e}^{n} x_{i} \alpha_{i j}=-\sum_{i=1}^{e-1} x_{i} \alpha_{i j} \in F .
$$

Therefore, for any $j, \sum_{i=e}^{n} \overline{x_{i}} \alpha_{i j}=0$, where $\overline{x_{i}}=x_{i}+F$. Hence

$$
\left[\overline{x_{e}} \cdots \overline{x_{n}}\right] \psi=0 \text {. }
$$

On the other hand, let $u_{1}, \ldots, u_{n-e} \in R^{\prime \prime n-e+1}$ be the columns of $\psi$ and consider the map $\theta: \bigwedge^{1} R^{\prime \prime n-e+1} \rightarrow \bigwedge^{n-e+1} R^{\prime \prime n-e+1} \simeq R^{\prime \prime}$ given by $x \mapsto x \wedge u_{1} \wedge \cdots \wedge u_{n-e}$. We have, by Lemma 5.1 ,

Moreover,

$$
\operatorname{im} \theta=I_{n-e}(\psi)
$$

$$
x \in \operatorname{im} \psi \Longleftrightarrow x \in\left\langle u_{1}, \ldots, u_{n-e}\right\rangle \Longrightarrow x \wedge u_{1} \wedge \cdots \wedge u_{n-e}=0 \Longleftrightarrow x \in \operatorname{ker} \theta,
$$

proving that $\operatorname{im} \psi \subseteq \operatorname{ker} \theta$. Therefore, there exists a complex of finite free $R^{\prime \prime}$-modules

$$
0 \rightarrow R^{\prime \prime n-e} \stackrel{\psi}{\rightarrow} R^{\prime \prime n-e+1} \stackrel{\theta}{\rightarrow} R^{\prime \prime} \rightarrow 0
$$


with $\operatorname{im} \theta=I_{n-e}(\psi) \subset R^{\prime \prime}$.

Now, suppose in addition that $E$ satisfies grade $F_{e}(E) \geq 2$. In this case, as we already observed, $\bar{E}=E^{\prime \prime} / F$ is a finitely generated torsionfree $R^{\prime \prime}$-module having rank 1. Hence, $\bar{E} \simeq I_{U}(E)=I$ a generic Bourbaki ideal of $E$ with respect to $U$. Since $I_{n-e}(\psi)=\left\langle\left|\psi_{e}\right|, \ldots,\left|\psi_{n}\right|\right\rangle$, where $\psi_{i}$ is obtained from $\psi$ by deleting the $(i-e+1)$-th row $(i=e, \ldots, n)$, and $I=\left\langle\overline{x_{e}}, \ldots, \overline{x_{n}}\right\rangle$ the mapping $\overline{x_{i}} \mapsto(-1)^{i-e}\left|\psi_{i}\right|(i=e, \ldots, n)$ extends to an $R^{\prime \prime}$-epimorphism $\rho: I \rightarrow I_{n-e}(\psi)$. In fact, if $\sum_{i=e}^{n} \beta_{i} \overline{x_{i}}=0$ and writing $\psi^{\prime}=\left[\begin{array}{cc}\beta_{e} & \\ \vdots & \psi \\ \beta_{n} & \end{array}\right]$ then $\left[\overline{x_{e}} \cdots \overline{x_{n}}\right] \psi^{\prime}=0$, and we deduce that $\overline{x_{i}}\left|\psi^{\prime}\right|=0,(i=e, \ldots, n)$.

Since $I=\left\langle\overline{x_{e}}, \ldots, \overline{x_{n}}\right\rangle, I\left|\psi^{\prime}\right|=0$ and so $\left|\psi^{\prime}\right| \in \operatorname{ann}_{R^{\prime \prime}}(I)$. But rank $I=1>0$, hence $\operatorname{ann}_{R^{\prime \prime}}(I)=0$. Therefore

$$
0=\left|\psi^{\prime}\right|=\sum_{i=e}^{n} \beta_{i}(-1)^{i-e}\left|\psi_{i}\right|,
$$

(by Laplace's theorem) proving that $\overline{x_{i}} \mapsto(-1)^{i-e}\left|\psi_{i}\right|$ is well defined. Hence there exists an $R^{\prime \prime}$-epimorphism $I \stackrel{\rho}{\rightarrow} I_{n-e}(\psi)$. Moreover, since $I, I_{n-e}(\psi)$ are $R^{\prime \prime}$-ideals of rank 1 (and every ideal is a torsionfree $R^{\prime \prime}$-module), $\operatorname{ker} \rho=0$ and we have

$$
I \simeq I_{n-e}(\psi) .
$$

Now, suppose that grade $I_{n-e}(\psi) \geq 2$. By Hilbert-Burch Theorem ([2, Theorem 1.4.17]), $I_{n-e}(\psi)$ has the free resolution

$$
0 \rightarrow R^{\prime \prime n-e} \stackrel{\psi}{\rightarrow} R^{\prime \prime n-e+1} \stackrel{\theta}{\rightarrow} I_{n-e}(\psi) \rightarrow 0 .
$$

Moreover, $I_{n-e}(\psi) \simeq R^{\prime \prime}$ or $I_{n-e}(\psi)$ is perfect of grade 2 . On the other hand, by Eq. (5.4),$I_{n-e}(\psi) \simeq I_{U}(E)=I$ a generic Bourbaki ideal of $E$ with respect to $U$ $\left(I_{U}(E) \simeq E^{\prime \prime} / F\right)$. If $E$ is not free, the Bourbaki ideal $I \simeq\left\langle\overline{x_{e}}, \ldots, \overline{x_{n}}\right\rangle$ is also not free (by Theorem 4.4). As a consequence, $I$ has a free resolution

$$
0 \rightarrow R^{\prime \prime n-e} \stackrel{\psi}{\rightarrow} R^{\prime \prime n-e+1} \rightarrow I \rightarrow 0,
$$

and we get that $I=I_{n-e}(\psi)$ is perfect of grade 2. Hence $\psi$ defines a presentation of $I$, with respect to $\overline{x_{e}}, \ldots, \overline{x_{n}}$.

We have proved the following result.

Theorem 5.3. Let $R$ be a Noetherian local ring, $E$ be a finitely generated $R$-module having rank $e \geq 2$ and $U$ a reduction of $E$. Let $R^{\prime \prime}, E^{\prime \prime}$ and $F=\oplus_{i=1}^{e-1} R^{\prime \prime} x_{i}$ as before. Let $x_{1}, \ldots, x_{n}$ be a generating set of $E^{\prime \prime}$ containing the basis $x_{1}, \ldots, x_{e-1}$ of $F$. Let $\varphi$ be an $n \times m$ matrix presenting $E^{\prime \prime}$ with respect to the generators $x_{1}, \ldots, x_{n}$ and let $\psi$ be an $(n-e+1) \times(n-e)$ submatrix of $\varphi$ satisfying grade $I_{n-e}(\psi) \geq 1$. Then there exists a complex of the form

$$
0 \rightarrow R^{\prime \prime n-e} \stackrel{\psi}{\rightarrow} R^{\prime \prime n-e+1} \rightarrow I_{n-e}(\psi) \rightarrow 0
$$


Suppose in addition that $E$ satisfies grade $F_{e}(E) \geq 2$. Then $E^{\prime \prime} / F$ is isomorphic to an $R^{\prime \prime}$-ideal $I=I_{U}(E)$ (a generic Bourbaki ideal of $E$ with respect to $U$ ) and

$$
I \simeq I_{n-e}(\psi) .
$$

Moreover, if $E$ is not free and grade $I_{n-e}(\psi) \geq 2$ then $I$ is perfect of grade 2 with a finite free resolution

$$
0 \rightarrow R^{\prime \prime n-e} \stackrel{\psi}{\rightarrow} R^{\prime \prime n-e+1} \rightarrow I \rightarrow 0
$$

and $\psi$ defines a presentation of $I$ with respect to the generators $\overline{x_{e}}, \ldots, \overline{x_{n}}$, with $I=I_{n-e}(\psi)$.

Corollary 5.4. Let $R$ be a Noetherian local ring, $E$ an ideal module having rank $e \geq 2$ and $U$ a reduction of $E$. Then any generic Bourbaki ideal of $E$ with respect to $U$ is isomorphic to a Fitting ideal.

Proof. Let $I=I_{U}(E) \subset R^{\prime \prime}$ be a generic Bourbaki ideal of $E$ with respect to $U$. Since grade $F_{e}(E) \geq 2$ then $I \simeq I_{n-e}(\psi)$, where $\psi$ is a submatrix of a matrix $\varphi$ presenting $E^{\prime \prime}$ (by the theorem above), as required.

For certain modules generic Bourbaki ideals can be chosen to have grade $\geq 2$, and we called them good generic Bourbaki ideals (see [4]). In fact, it is proved in [18, Proposition 3.2] that a finitely generated $R$-module $E$ having rank $e>0$ and satisfying grade $F_{e}(E) \geq 2$ has a good generic Bourbaki ideal if and only if $E$ is orientable. In particular, ideal modules also have good generic Bourbaki ideals. As already observed, $V\left(F_{e}(E)\right)=\operatorname{Supp} G / E=\operatorname{Supp} R / F_{e}(E)$ in the case where $E \subseteq G \simeq R^{e}$ is an ideal module. In particular, $V\left(F_{1}(I)\right)=\operatorname{Supp} R / I=V(I)$ for any $R$-ideal $I$ with grade $I \geq 2$. Moreover, if $I \simeq E^{\prime \prime} / F$ is a good generic Bourbaki ideal of $E$ then, clearly,

$$
V\left(F_{e}\left(E^{\prime \prime}\right)\right) \subseteq V\left(F_{1}(I)\right)=V(I) .
$$

The following result characterizes ideal modules with projective dimension equal to one via generic Bourbaki ideals. Using the notations of Theorem [5.3, this shows in particular that if $E$ is not free and grade $I_{n-e}(\psi) \geq 2$ then proj dim $E=1$.

Proposition 5.5. Let $R$ be a Noetherian local ring, $E$ an ideal module having rank $e \geq 2$ and $U$ a reduction of $E$. Then the following are equivalent:

a) $\operatorname{proj} \operatorname{dim} E=1$.

b) Any good generic Bourbaki ideal of $E$ (with respect to $U$ ) is perfect of grade 2.

c) There exists a generic Bourbaki ideal of $E$ (with respect to $U$ ) which is perfect of grade 2 .

Proof. Suppose that $\bar{E} \simeq I_{U}(E)=I$ is an $R^{\prime \prime}$-ideal. By Theorem 4.4

$$
\text { proj } \operatorname{dim} R^{\prime \prime} / I=\operatorname{proj} \operatorname{dim} \bar{E}+1=\operatorname{proj} \operatorname{dim} E+1 .
$$

Since grade $I \leq \operatorname{proj} \operatorname{dim} R^{\prime \prime} / I$ the equivalences then follow. 
Remark 5.6. Let $I$ be a perfect ideal of grade 2. Then, ht $I \leq 2$ by [20, Theorem 1.1.18], and because grade $I \leq$ ht $I$ we have that ht $I=2$. This can be extended to modules of projective dimension 1 in the following way: Let $E$ be a finitely generated $R$-module having rank $e$. Suppose that proj dim $E=1$. Then ht $F_{e}(E) \leq e+1$.

Proof. Let $0 \rightarrow R^{m} \stackrel{\varphi}{\rightarrow} R^{n} \rightarrow E \rightarrow 0$ be a (minimal) free resolution of $E$. Since $\operatorname{rank} E=e$ then $m=n-e$. Moreover, $I_{n-e}(\varphi) \neq 0$ and $I_{n-e+1}(\varphi)=0$. Thus, by [20, Theorem 1.1.18],

$$
\text { ht } F_{e}(E)=\text { ht } I_{n-e}(\varphi) \leq e+1
$$

with $m=n-e=t$.

In [3] we defined the analytic deviation, for an ideal module $E \subsetneq G \simeq R^{e}$ with positive rank $e>0$, as ad $(E)=\ell(E)-e+1-$ ht $F_{e}(E)$. We say that $E$ is equimultiple if $\operatorname{ad}(E)=0$. Moreover, we say that $E$ is a module of the principal class if $\mu(E)=$ ht $F_{e}(E)+e-1$ and that $E$ is a complete intersection if $\mu(E)=$ grade $F_{e}(E)+e-1$. It is clear that these notions agree with the correspondent ones for ideals. Moreover, since $\mu(E) \geq \ell(E)$ (by Eq. (3.2)),$\ell(E) \geq$ ht $F_{e}(E)+e-1$ (by [3, Proposition 3.12]), and grade $F_{e}(E)=$ grade $G / E \geq 2$, then

$$
\mu(E) \geq \ell(E) \geq \operatorname{ht} F_{e}(E)+e-1 \geq \operatorname{grade} F_{e}(E)+e-1 \geq e+1 .
$$

Suppose that $I$ is a good generic Bourbaki ideal of $E$. Since $I \simeq E^{\prime \prime} / F$ we always have $\mu(E) \leq \mu(I)+e-1$. On the other hand, from Eq. (5.5) we have that ht $I \leq$ ht $F_{e}\left(E^{\prime \prime}\right)=$ ht $F_{e}(E)$. Hence, if $I$ is an ideal of the principal class [complete intersection or equimultiple, respectively] then $E$ satisfies the same property. However we are interested in the other situation, i.e. to know when a generic Bourbaki ideal of $E$ satisfies the same property as the module $E$. If $\operatorname{dim} R=2$ this is always true, since then $E$ is of the principal class if and only if $\mu(E)=e+1$ and so by Theorem 4.4 we have $\mu(I)=2=\operatorname{ht}(I)$ (note that $R$ must be Cohen-Macaulay and so to be of the principal class is the same as to be a complete intersection). And similarly for the equimultiple property by using the equality (4.2). In fact, in the general case case where $\mu(E)$ or $\ell(E)$ reach the minimum values we have the following result.

Proposition 5.7. Let $(R, \mathfrak{m}, k)$ be a Noetherian local ring and let $E \subsetneq G \simeq R^{e}$ be an ideal module having rank $e>0$.

a) If $\mu(E)=e+1$ then $E$ is a module of the principal class.

b) If $\ell(E)=e+1$ and $k$ is infinite then $E$ is an equimultiple module.

In both cases, if $I$ is a good generic Bourbaki ideal of $E$ then I satisfies the same properties as $E$ and we have

$$
\text { grade } F_{e}(E)=\text { ht } F_{e}(E)=2=\text { ht } I=\text { grade } I \text {. }
$$

Proof. (a) If $\mu(E)=e+1$ then $\ell(E)=e+1$ (by Eq. (5.6) ). Hence

$$
2 \leq \operatorname{grade} F_{e}(E) \leq \operatorname{ht} F_{e}(E) \leq \mu(E)-e+1=2
$$

and so

$$
\mu(E)=\ell(E)=\operatorname{ht} F_{e}(E)+e-1=\operatorname{grade} F_{e}(E)+e-1,
$$


proving that $E$ is of the principal class.

(b) Let $U$ be a minimal reduction of $E$. Then $\mu(U)=\ell(E)=e+1$. By (a), $U$ is of the principal class. Then $E$ is an equimultiple module (by [3, Proposition 4.3]) and we have

$$
\text { grade } F_{e}(E)=\text { ht } F_{e}(E)=2 \text {. }
$$

Now, let $I$ be a good generic Bourbaki ideal of $E$. Then, using Eq. (4.2) and (4.5), we get for (a)

$$
\mu(I)=2=\ell(I), 2 \leq \text { grade } I \leq \text { ht } I \leq \ell(I)=2
$$

and so

$$
\mu(I)=\ell(I)=\text { ht } I=\text { grade } I=2 .
$$

Therefore, $I$ is of the principal class. In the same way, supposing that $\ell(E)=e+1$, then $\ell(I)=2$, and so

$$
\ell(I)=\text { ht } I=\text { grade } I=2 .
$$

Therefore, $I$ is an equimultiple ideal. In both cases, the equalities (5.7) hold.

\section{Divisors of A MODUle - PART 2}

So far we have proved in Section 2 that if $E$ is a finitely generated $R$-module with rank $e$, and that if

$$
R^{m} \stackrel{\varphi}{\rightarrow} R^{n} \stackrel{\phi}{\rightarrow} E \rightarrow 0
$$

is a finite presentation of $E$, then

$$
\operatorname{det}_{0}(E) \simeq[[E]] \simeq I_{n-e}(\rho)=F_{e}\left(E_{1}\right) \subseteq F_{e}(E),
$$

where $\rho$ is an $n \times(n-e)$ submatrix of $\varphi$ with a non-zero $(n-e) \times(n-e)$ minor, and $E_{1}$ is an $R$-module of projective dimension 1 with rank $e$ with a finite presentation given by $\rho$. Moreover, if $E \subseteq G \simeq R^{e}$ then

$$
F_{0}(G / E)=\operatorname{det}_{0}(E) .
$$

Now, we want to include in this context the Bourbaki ideal. Since we cannot do this directly over the ring $R$, we have to extend it previously to an adequate Nagata extension $R^{\prime \prime}$. And this inclusion will be possible by means of the norm ideal $[[E]]_{R}$ and the fact proven in the previous section that any generic Bourbaki ideal is always isomorphic to a Fitting ideal.

We begin by observing that the norm ideal behaves well under the extension of scalars by flat homomorphisms.

Proposition 6.1. Let $h: R \rightarrow S$ be a flat homomorphism of rings. Then

$$
\left[\left[E \otimes_{R} S\right]\right]_{S} \simeq[[E]]_{R} \otimes_{R} S .
$$

Proof. It is known that if $E$ has rank and $h: R \rightarrow S$ is flat, then $E \otimes_{R} S$ has rank and $\operatorname{rank}_{R} E=\operatorname{rank}_{S} E \otimes_{R} S$. Then, the statement follows by Eq. (2.11) and Properties 2.1 . 
In particular, supposing that $R^{\prime \prime}$ is a Nagata extension of $R$ and $E^{\prime \prime}=E \otimes_{R} R^{\prime \prime}$, as in the previous sections, we get

$$
\left[\left[E^{\prime \prime}\right]\right]_{R^{\prime \prime}}=\left[\left[E \otimes_{R} R^{\prime \prime}\right]\right]_{R^{\prime \prime}} \simeq[[E]]_{R} \otimes_{R} R^{\prime \prime}=\operatorname{det}_{0}(E) \cdot R^{\prime \prime} .
$$

In the notation of section 4, suppose that $U=\sum_{i=1}^{k} R a_{i}$ is a reduction of $E$ and $F=\sum_{j=1}^{e-1} R^{\prime \prime} x_{j}$ with $x_{j}=\sum_{i=1}^{k} z_{i j} a_{i}$. Let $\left\{x_{1}, \ldots, x_{n}\right\}$ be a generating set of $E^{\prime \prime}$ containing the basis $\left(x_{1}, \ldots, x_{e-1}\right)$ of $F$. Let

$$
R^{\prime \prime m} \stackrel{\varphi}{\rightarrow} R^{\prime \prime n} \rightarrow E^{\prime \prime} \rightarrow 0
$$

be a finite free presentation of $E^{\prime \prime}$ with respect to the generators $x_{1}, \ldots, x_{n}$, and write also by $\varphi$ the respective relation matrix. As we observed, in section 5 , there exists an $(n-e+1) \times(n-e)$ submatrix $\psi$ of $\varphi$ satisfying grade $I_{n-e}(\psi) \geq 1$. Moreover, if grade $F_{e}(E) \geq 2$ then $I_{n-e}(\psi) \simeq E^{\prime \prime} / F \simeq I$ (Theorem 5.3) with $I$ a generic Bourbaki ideal of $E$. Now let $\rho$ be the $n \times(n-e)$ submatrix of $\varphi$ containing $\psi$. The matrix $\rho$ defines a finite free presentation

$$
0 \rightarrow R^{\prime \prime n-e} \stackrel{\rho}{\rightarrow} R^{\prime \prime n} \rightarrow E_{1}^{\prime \prime} \rightarrow 0
$$

with $E_{1}^{\prime \prime}$ an $R^{\prime \prime}$-module satisfying $\operatorname{rank}\left(E_{1}^{\prime \prime}\right)=e, E_{1}^{\prime \prime} / \tau_{R^{\prime \prime}}\left(E_{1}^{\prime \prime}\right) \simeq E^{\prime \prime}$ (as observed in section 2). Therefore

$$
I_{n-e}(\psi) \subseteq I_{n-e}(\rho) \subseteq I_{n-e}(\varphi)
$$

Moreover, by Eq. (6.1)

$$
\operatorname{det}_{0}(E) \cdot R^{\prime \prime} \simeq\left[\left[E^{\prime \prime}\right]\right]_{R^{\prime \prime}} \simeq F_{e}\left(E_{1}^{\prime \prime}\right) \subseteq F_{e}\left(E^{\prime \prime}\right) \simeq F_{e}(E) \otimes_{R} R^{\prime \prime}=F_{e}(E) \cdot R^{\prime \prime} .
$$

Further, if grade $F_{e}(E) \geq 2$ then

$$
I \simeq I_{n-e}(\psi) \subseteq I_{n-e}(\rho)=F_{e}\left(E_{1}^{\prime \prime}\right) \simeq\left[\left[E^{\prime \prime}\right]\right]_{R^{\prime \prime}} .
$$

In the case where $E$ has projective dimension equal to one, we may assert in addition that $F_{e}\left(E^{\prime \prime}\right)$ is a representative of $\left[\left[E^{\prime \prime}\right]\right]_{R^{\prime \prime}}$.

Proposition 6.2. Let $R$ be a Noetherian local ring and $E$ a finitely generated $R$ module having rank $e \geq 2$. If grade $F_{e}(E) \geq 2$ then

$$
I \simeq I_{n-e}(\psi) \subseteq I_{n-e}(\rho) \subseteq F_{e}\left(E^{\prime \prime}\right),
$$

with $I_{n-e}(\rho) \simeq\left[\left[E^{\prime \prime}\right]\right]_{R^{\prime \prime}} \simeq \operatorname{det}_{0}(E) \cdot R^{\prime \prime}$, for any generic Bourbaki ideal $I$ of $E$. If moreover proj $\operatorname{dim} E=1$, then

$$
I \simeq I_{n-e}(\psi) \subseteq I_{n-e}(\rho)=F_{e}\left(E^{\prime \prime}\right)
$$

for any generic Bourbaki ideal I of E.

Proof. Follows by Eq. (6.2) and (6.4).

Next suppose that $(R, \mathfrak{m})$ is a 2 -dimensional regular local ring and $E$ is a finitely generated torsionfree $R$-module with rank $e$. Recall that $E$ is said to be contracted if $E=E S \cap R^{e}$, where $S=R\left[\frac{\mathfrak{m}}{a}\right], a$ is a minimal generator of $\mathfrak{m}$ and $E S$ is the $S$ submodule of $S^{e}$ generated by $S$. Contracted modules were defined by V. Kodiyalam in [7] as an extension to modules of the notion of contracted ideal. Integrally closed modules over a 2-dimensional regular local ring $R$ are contracted. Given a contracted 
module $E$, it may be seen that $G=E^{* *}$ is free and that $G / E$ is of finite length, so grade $G / E=2$ and $E$ is an ideal module, see [7, Proposition 2.1].

It then happens that contracted modules have a special generic Bourbaki ideal: It is proved in [6, Corollary 3.6] that if $E$ is contracted then $F_{e}\left(E^{\prime \prime}\right)$ is a good generic Bourbaki ideal. So as a consequence of all the above relations we have the following:

Corollary 6.3. Let $(R, \mathfrak{m})$ be a 2-dimensional regular local ring and $E$ a finitely generated torsionfree $R$-module with rank $e$ which is not free. If $E$ is contracted then there exists a good generic Bourbaki ideal $I$ of $E$ which is a representative of $\left[\left[E^{\prime \prime}\right]\right]_{R^{\prime \prime}}$ and in this case

$$
I=F_{e}\left(E^{\prime \prime}\right) \simeq\left[\left[E^{\prime \prime}\right]\right]_{R^{\prime \prime}} \simeq \operatorname{det}_{0}(E) \cdot R^{\prime \prime} .
$$

Proof. Since $E$ is an ideal module, grade $F_{e}(E)=2$. Also, proj $\operatorname{dim} E=1$. The result then follows by Proposition 6.2.

We finish by pointing out that, in the above conditions, the blow up at any generic Bourbaki ideal has an universal flattening property under birational morphisms, as we described in Theorem 2.10. For instance, if $E$ is an integrally closed module over a 2-dimensional regular local ring $R$, we have that the Rees algebra of $E$ is Cohen-Macaulay. So after a suitable Nagata extension $R^{\prime \prime}$ of $R$, this Rees algebra is a deformation of the Rees algebra of a good generic Bourbaki ideal of $E$, whose blow up has such universal flattening property with respect to $E^{\prime \prime}=E \otimes_{R} R^{\prime \prime}$. It would be interesting to know other instances where the Rees algebra of a module satisfies a similar universal property.

\section{REFERENCES}

[1] N. Bourbaki, Algèbre Commutative, Chapters I-VII, Hermann, Paris, 1961-1967.

[2] W. Bruns \& J. Herzog, Cohen-Macaulay rings, Cambridge Studies in Advanced Mathematics 39, Cambridge University Press, Cambridge, 1998.

[3] A. L. Branco Correia \& S. Zarzuela, On Equimultiple Modules, Communications in Algebra 37, Issue 6, (2009), 1949-1976.

[4] A. L. Branco Correia \& S. Zarzuela, On the depth of the Rees algebra of an ideal module, J. of Algebra 323, Issue 5, (2010), 1503-1529.

[5] N. Hara \& T. Sawada \& T. Yasuda, F-Blowups of Normal Surface Singularities, arXiv:math/1108.1840v2, 2011.

[6] J. Hong \& B. Ulrich, Specialization and Integral Closures, arXiv:math/0611773v1, 2006.

[7] V. Kodiyalam, Integrally closed modules over two-dimensional regular local rings, Trans. Amer. Math. Soc 347 (1995), 3551-3573.

[8] E. Kunz, Introduction to Commutative Algebra and Algebraic Geometry, Birkhäuser Boston, 1985.

[9] E. Kunz, Kähler Differentials, Advanced Lectures in Mathematics, Fried. Vieweg \& Sohn, 1986.

[10] D. R. Grayson \& M. E. Stillman, Macaulay2, a software system for research in algebraic geometry, available at http://www.math.uiuc.edu/Macaula2/, J. of Algebra 197 (1997), 311-341.

[11] A. Micali, Sur les algèbres de Rees, Bull. Soc. Math. Belg., 20 (1968), 215-235.

[12] A. Oneto \& E. Zaniti, Remarks of Nash Blowing-up, Rend. Sem. Mat. Univ. Pol. Torino 49 (1991), 71-82. 
[13] A. Ooishi, Reductions of graded rings and pseudo-flat graded modules, Hiroshima Math. J., 18 (1988), 463-477.

[14] D. Rees, Reductions of modules, Math. Proc. Camb. Phil. Soc. 101 (1987), 431-449.

[15] H. Rossi, Picdard variety of an isolated singular point, Rice University Studies 54 (1968), 63-73.

[16] A. Simis, K. Smith, B. Ulrich An algebraic proof of Zak's inequality for the dimension of the Gauss image, Math. Z. 241 (2002) 871-881.

[17] A. Simis, B. Ulrich The Fitting Ideal Problem, Bull. London Math. Soc. (2009) 41 (1), $79-88$.

[18] A. Simis, B. Ulrich, W. Vasconcelos, Rees algebras of modules, Proc. London Math. Soc. 87 (3) (2003), 610-646.

[19] I. Swanson \& C. Huneke, Integral Closure of Ideals, Rings, and Modules, London Mathematical Society, Lecture Note Series 336, Cambridge University Press, 2006.

[20] W. Vasconcelos, Arithmetic of Blowup Algebras, London Mathematical Society Lecture Notes Series, 195, Cambridge University Press, 1994.

[21] W. Vasconcelos, Integral Closure, Springer Monographs in Mathematics, 2005.

[22] O. Villamayor, On flattening of coherent sheaves and of projective morphisms, J. Algebra 295 (2006), 119-140.

[23] T. Yasuda, Higher Nash blowups, Compositio Math. 143 (2007), 1493-1510.

[24] T. Yasuda, Universal Flattening of Frobenius, American Journal of Mathematics 134 (2012), 349-378.

Centro de Estruturas Lineares e Combinatórias, Universidade de Lisboa, Av. Prof. Gama Pinto 2, 1649-003 Lisboa, Portugal

E-mail address: alcorreia@cii.fc.ul.pt

Universidade Aberta, Departamento de Ciências e Tecnologia, Palácio Ceia, Rua Da Escola Politécnica, 147, 1269-001 Lisboa, Portugal

E-mail address: matalrbc@uab.pt

Departament d’Àlgebra i Geometria, Universitat de Barcelona, Gran Via 585, E-08007 BARCELONA, SpAin

E-mail address: zarzuela@mat.ub.es 